\title{
Staphylococcus aureus Second Immunoglobulin-Binding Protein drives atopic dermatitis via IL-33
}

DOI:

10.1016/j.jaci.2020.09.023

\section{Document Version}

Accepted author manuscript

Link to publication record in Manchester Research Explorer

\section{Citation for published version (APA):}

Al Kindi, A., Williams, H., Matsuda, K., Alkahtani, A., Saville, C., Bennett, H., Alshammari, Y., Tan, S. Y., O'Neill, C., Tanaka, A., Matsuda, H., Arkwright, P., \& Pennock, J. (2021). Staphylococcus aureus Second ImmunoglobulinBinding Protein drives atopic dermatitis via IL-33. Journal of Allergy and Clinical Immunology, 147(4), 13541368.e3. [0]. https://doi.org/10.1016/j.jaci.2020.09.023

\section{Published in:}

Journal of Allergy and Clinical Immunology

\section{Citing this paper}

Please note that where the full-text provided on Manchester Research Explorer is the Author Accepted Manuscript or Proof version this may differ from the final Published version. If citing, it is advised that you check and use the publisher's definitive version.

\section{General rights}

Copyright and moral rights for the publications made accessible in the Research Explorer are retained by the authors and/or other copyright owners and it is a condition of accessing publications that users recognise and abide by the legal requirements associated with these rights.

\section{Takedown policy}

If you believe that this document breaches copyright please refer to the University of Manchester's Takedown Procedures [http://man.ac.uk/04Y6Bo] or contact uml.scholarlycommunications@manchester.ac.uk providing relevant details, so we can investigate your claim.

\section{OPEN ACCESS}


1 Staphylococcus aureus Second Immunoglobulin-Binding Protein drives atopic

2 dermatitis via IL-33

3 Arwa Al Kindi, PhD, ${ }^{1 \dagger}$ Helen Williams, PhD, ${ }^{1 \dagger}$ Kenshiro Matsuda, PhD, ${ }^{2}$ Abdullah M.

4 Alkahtani, PhD, ${ }^{3}$ Charis Saville, PhD, ${ }^{1}$ Hayley Bennett, $\mathrm{PhD},{ }^{4}$ Yasmine Alshammari, MSc, ${ }^{1}$

5 Soo Y. Tan, PhD, ${ }^{5}$ Catherine O'Neill, PhD, ${ }^{6}$ Akane Tanaka, DVM, PhD, ${ }^{7}$ Hiroshi Matsuda,

6 DVM, PhD, ${ }^{8}$ Peter D. Arkwright, MD, PhD,${ }^{1 *}{\text { Joanne L. Pennock, } \text { PhD }^{1 *}}^{*}$

$7 \quad{ }^{1}$ Lydia Becker Institute of Immunology and Inflammation, University of Manchester, United

8 Kingdom, ${ }^{2}$ Center for Innovative Drug Discovery, University of Tsukuba, Japan, ${ }^{3}$ Department of

9 Medicine, Microbiology and Parasitology, King Khalid University, Abha, Saudi Arabia,

${ }^{4}$ Genome Editing Unit, University of Manchester, United Kingdom, ${ }^{5}$ National University Health

11 System, Singapore, ${ }^{6}$ Division of Dermatological and Musculoskeletal Sciences, University of

12 Manchester, United Kingdom. ${ }^{7}$ Laboratories of Veterinary Molecular Pathology \& Therapeutics

13 and ${ }^{8}$ Comparative Animal Medicine, Tokyo University of Agriculture \& Technology, Tokyo,

14 Japan

$15 t, *$ authors contributed equally to this work.

16 Corresponding author: Dr P D Arkwright, Lydia Becker Institute of Immunology \&

17 Inflammation, University of Manchester, Room 2.21, Core Technology Facility, 46 Grafton St.,

18 Manchester, M13 9NT, United Kingdom. Telephone +44 161306 3771, email

19 peter.arkwright@manchester.ac.uk

20 Declaration of Funding This research was supported by Leo Pharma (Project Grant No.

21 LF16080). Dr Arwa Al Kindi received a scholarship from the Government of the Sultanate of

22 Oman. The Grant-in-Aid for Scientific Research grants Start-up \#17H06669 (to K. Matsuda), S

23 \#16H06383 (to H. Matsuda), and A \#19H00969 and Fostering Joint International Research B 
24 \#18KK0191 (to A. Tanaka), provided by the Japan Society for the Promotion of Science. The

25 University of Manchester Flow cytometry core facility-flow cytometers used in this study were

26 purchased with grants from BBSRC, Wellcome and the University of Manchester Strategic Fund.

27

28 Conflict of interests None of the authors declare any competing financial and/or non-financial

29 interests in relation to the work described.

30 Word count (excluding Abstract, Figures/Table legends): 4,764 
Abstract

Background: S. aureus is the dominant infective trigger of atopic dermatitis (AD). How this bacterium drives type 2 allergic pathology in the absence of infection in AD patients is unclear.

Objective: To identify the $S$. aureus-derived virulence factor(s) that initiates the cutaneous type 2-promoting immune response responsible for AD. initiating the type-2 promoting cytokine activity underlying AD.

Methods: In vitro human keratinocyte cell culture, ex vivo human skin organ explants and the eczema prone $\mathrm{Nc}$ /Tnd mouse were used as model systems to assess type-2 promoting immune responses to $S$. aureus. Identification of the bioactive factor was accomplished using Fast Protein Liquid Chromatography and mass spectrometry. Bioactivity was confirmed by cloning and expression in an $E$. coli vector system, and $S$. aureus Sbi mutant strains confirming loss of activity.

Results: $S$. aureus was unique amongst staphylococcal species in its ability to induce the rapid release of constitutive IL-33 from human keratinocytes independent of the toll-like receptor pathway. Using the eczema-prone NC/Tnd mouse model, we showed that IL-33 was essential in inducing the immune response to $S$. aureus in vivo. By fractionation and candidate testing, we identified the Second Immunoglobulin-Binding Protein (Sbi) as the predominant staphylococcus-derived virulence factor that directly drives IL-33 release from human keratinocytes. Immunohistology of skin demonstrated that corneodesmosin, a component of corneodesmosomes that form key intercellular adhesive structures in the stratum corneum, was disrupted resulting in reduction of skin barrier function.

Conclusion: S. aureus-derived Sbi is a unique type 2-promoting virulence factor capable of 
Clinical Implications: The Second Immunoglobulin-Binding Protein (Sbi) produced solely by $S$. aureus species, is an important factor inducing type-2 promoting cytokine responses and atopic dermatitis.

Capsule summary: Why $S$. aureus is the predominant infective trigger of AD is unclear. This study identifies $S$. aureus derived Sbi as a key virulence factor, capable of triggering type 2 immune responses in vitro and in vivo.

Key words: Staphylococcus aureus, atopic dermatitis, Second Immunoglobulin-Binding Protein, Sbi, virulence factor, skin, keratinocytes, IL-33, TSLP, type 2 immune response.

Abbreviations: AD: atopic dermatitis; CDSN: corneodesmosin; CFU: Colony Forming Units; DAPI: 4',6-diamidino-2-phenylindole; DSC-1: desmocollin-1; DSG-1: desmoglein-1; DF: Dermatophagoides farinae; DLD: DihydroLipoyl Dehydrogenase; DP: Dermatophagoides pteronyssinus; DPBS: Dulbecco's Phosphate Buffered Saline; ELISA: Enzyme-Linked Immunosorbent Assay; FITC: Fluorescein isothiocyanate; fnb: fibronectin; FPLC: Fast protein liquid chromatography; FLG: filaggrin; FSA: Filtered S. aureus supernatant; FSE: Filtered $S$. epidermidis supernatant; HEKa: Human Epithelial Keratinocytes, adult: HKSA: Heat-killed S. aureus; IL: interleukin; LAP: Leucine AminoPeptidase; LiSA: live S. aureus; LPS:

lipopolysaccharide; LTA: lipoteichoic acid; MSM/Ms: Mus musculus molossinus/Mishima strain; NC/Tnd: Nishiki-nezumi Cinnamon/Tokyo University of Agriculture and Technology strain; NHEK: Normal Human Epidermal Keratinocytes; PDHa: Pyruvate Dehydrogenase Alphasubunit; PGN: proteoglycan; Pam ${ }_{3} \mathrm{CSK}_{4}$ : N-Palmitoyl-S-[2,3-bis(palmitoyloxy)-(2RS)-propyl][R]-cysteinyl-[S]-seryl-[S]-lysyl-[S]-lysyl-[S]-lysyl-[S]-lysine; RT: room temperature; Sbi: Second Immunoglobulin-Binding Protein; SCORAD: scoring AD; SDS-PAGE: sodium dodecyl sulphate-polyacrylamide gel electrophoresis, SEM: Standard Error of the Mean; siRNA: small 
81 interfering RNA; Spl: Serine-protease-like; TEWL: TransEpidermal Water Loss; TLR: Toll-like 82 receptor; TSLP: thymic stromal lymphopoietin. 

majority of skin and soft tissue infections worldwide. ${ }^{1-3}$ It is also the most frequent cause of infection-induced flares of atopic dermatitis (AD) ${ }^{4-6}$ The bacterium expresses many virulence factors both in its cell wall and secretome. ${ }^{7-9}$ Previous studies have focused on recognition of $S$. aureus by host innate immunity, particularly activation of the inflammasome through toll-like receptors (TLR) and subsequent induction of cytokines such as IL-1 $1 .{ }^{10}$ Enzymes such as coagulase, toxins such as enterotoxins, Toxic Shock Syndrome Toxin-1, $\alpha$-hemolysin, and invasins including pore-forming proteins such as Panton-Valentine Leukocidin induce host cytotoxicity and damage the skin barrier. Defects in skin barrier function, particularly lack of filaggrin expression in the epidermis secondary to pathologic variants in the $F L G$ gene, are well recognized to contribute to the pathogenesis of $\mathrm{AD} .{ }^{11}$ However, patients with $F L G$ variants may suffer from ichthyosis without skin inflammation. ${ }^{12}$ Thus although skin barrier disruption can predispose patients to $\mathrm{AD}$, the main driving force in $\mathrm{AD}$ is the Th2 immune response, as clearly illustrated by the effectiveness of the IL-4/13 receptor antagonist dupilumab in recent clinical trials. ${ }^{13,14}$ Deltatoxins and superantigens produced by $S$. aureus can induce mast cell activation and IgE production, but the bacterial trigger of type-2 allergic responses by keratinocytes in the skin is unknown. ${ }^{15,16}$ The current paradigm is that rather than a single factor, a complex array of $S$. aureus virulence factors contribute to the atopic skin response. ${ }^{4,7}$ However, the study of immunodeficiency and immune dysregulation disorders have taught us that there is much redundancy in host immunity. ${ }^{17,18}$ The hypothesis we set out to explore was whether S. aureus expresses a predominant virulence factor that initiates type 2-promoting cytokine release from skin cells and drives the development of $\mathrm{AD}$. If this critical factor could be found, then targeting it therapeutically could negate one of the key advantages $S$. aureus has developed during its evolution and symbiosis with its hosts. 


\section{METHODS}

\section{Materials and reagents}

A list of bacterial strains, materials, reagents, and primer sequences used in this study are listed in the online repository Tables SI and SII.

Staphylococcal species and strains, Group A streptococcus and preparation of filtered supernatants

114 Methicillin-sensitive S. aureus (LiSA) and Streptococcus pyogenes were provided by Professor

115 McBain. Other S. aureus wild-type strains and staphylococcal species were a gift from Dr Xia, 116 both of University of Manchester. S. aureus SH1000 and its isogenic fibronectin (fnb)A- and fnbB117 mutant, as well as the Newman strain, Newman $\mathrm{Sbi}^{-}, \mathrm{Spa}^{-}$, and $\mathrm{pRMC}: \mathrm{sbi}^{-}$mutants were a gift 118 from Professor Geoghegan, Trinity College, Dublin (refer to Online repository Table SI). Both the 119 Newman $\mathrm{Sbi}^{-}$and $\mathrm{pRMC}: \mathrm{sbi}^{-}$mutant strains inherently express no Sbi protein, but the $\mathrm{pRMC}^{-\mathrm{sbi}^{-}}$ 120 strain can be induced to express Sbi with addition of anhydrotetracyclines, although not in these

121 set of experiments. ${ }^{19}$ Staphylococcal species were plated overnight $\left(37^{\circ} \mathrm{C}\right)$ on nutrient agar to 122 generate colonies. The number of colony-forming units $(\mathrm{CFU} / \mathrm{ml})$ were determined by the method 123 of Miles \& Misra.

\section{Preparation of filtered supernatant from $S$. aureus and $S$. epidermidis}

A single bacterial colony was inoculated with nutrient broth overnight $\left(37^{\circ} \mathrm{C}\right) \cdot 10^{8} \mathrm{CFU} /$ species

127 were inoculated in $100 \mathrm{ml}$ of Human Keratinocyte Growth Medium 2 (PromoCell) and incubated 128 for $6 \mathrm{~h}$ at $37^{\circ} \mathrm{C}$ in a shaking incubator. After incubation, samples were centrifuged $(1,600 \mathrm{~g}, 5 \mathrm{~min}$, 129 RT), supernatants collected and filter sterilized using $0.2 \mu \mathrm{m}$ filters (Millipore, Bedford, USA). The 130 filtered supernatants were then treated with $2 \%$ penicillin/streptomycin and stored at $-80^{\circ} \mathrm{C}$ until 131 required for stimulation experiments. 
133 Preparation of modified allergens (ragweed and house dust mites)

134 Allergens used included modified Ragweed, Dermatophagoides farinae (DF) and 135 Dermatophagoides pteronyssinus (DP) (gifts from Dr Gianni Mistrello, Lofarma). The allergen 136 extracts were re-suspended in sterile Dulbecco's Phosphate Buffered Saline (DPBS) (Sigma137 Aldrich) according to supplier recommendations at a concentration of $6.0 \mathrm{mg} / \mathrm{ml}$ Ragweed, $1.3 \mathrm{mg} / \mathrm{ml} \mathrm{DF}$ and $2.6 \mathrm{mg} / \mathrm{ml} \mathrm{DP}$ were stored at $-20^{\circ} \mathrm{C}$ until required for stimulation experiments.

\section{Primary human keratinocyte and ex vivo organ culture and stimulation}

NHEK (PromoCell) and HEKa (ThermoFisher Scientific) were cultured in Keratinocyte Growth Medium 2 plus supplements (PromoCell) and medium 154 supplemented with Human Keratinocyte Growth Supplement without antibiotics respectively for $48-72 \mathrm{~h}$ at $37^{\circ} \mathrm{C}$ under $5 \%$ $\mathrm{CO}_{2}$ until $70-90 \%$ confluent. Cells of passages $3-6$ were used for experiments. Human keratinocytes were cultured in 24 -well tissue culture plates at a density of $5 \times 10^{5}$ cells/well. Primary human fibroblasts and keratinocytes were isolated from skin biopsies taken from either nonatopic patients with otapostasis undergoing plastic surgery or similarly aged children with moderatesevere AD attending a Regional Paediatric AD outpatient clinic at Royal Manchester Children's Hospital, Manchester, UK.

Human skin was obtained from adult healthy patients following abdominal reduction or liposculpture procedures. Post-excision, subcutaneous fat tissue was removed, biopsies were taken using sterile 4mm biopsy punches (KAI Medical, GP Supplies Ltd, London, UK) and placed in 1ml/biopsy William's E medium (Thermo Fisher Scientific) supplemented with l-glutamine (2mM,

154 Sigma-Aldrich), penicillin (100U/ml)-streptomycin $(0.1 \mathrm{mg} / \mathrm{ml})$ (Sigma-Aldrich), 0.02\% (v/v) 155 hydrocortisone (Sigma-Aldrich) and 0.1\% (v/v) insulin (Sigma-Aldrich) in 6-well culture plates 156 containing $0.4 \mu \mathrm{m}$ ThinCert ${ }^{\mathrm{TM}}$ cell culture inserts (Griener Bio-One, Kremsmünster, Austria) 
dermal side down. Intact epidermal exposed biopsies were treated with $5 \mu 1 /$ biopsy of FSA, FSE or allergens including Ragweed, DF, and DP for up to $12 \mathrm{~h}\left(37^{\circ} \mathrm{C}, 5 \% \mathrm{CO}_{2}\right)$. Following treatments, 159 supernatant was removed and stored at $-80^{\circ} \mathrm{C}$, and biopsies were snap-frozen in liquid nitrogen and stored at $-80^{\circ} \mathrm{C}$ until use, where they were then embedded in Optimal Cutting Temperature Compound (KP Cryo-Compound) before cryo-sectioning.

\section{Human IL-33 and TSLP ELISA}

R\&D System ELISA (Abingdon, UK) were used to measure IL-33 (DY3625-05) and TSLP (DY1398-05) release according to the manufacturer's instructions.

\section{Detection of cell death by Annexin-V and DAPI staining}

Cells were detached from 24-well plates, harvested into micro-centrifuge tubes, washed with Annexin- $\mathrm{V}$ binding buffer and centrifuged $\left(5 \mathrm{~min}, 500 \mathrm{~g}, 4^{\circ} \mathrm{C}\right)$, then stained with Annexin- $\mathrm{V}$ diluted 1:100 in binding buffer for 30min on ice. Following incubation, cells were harvested and resuspended in Annexin-V binding buffer. DAPI was added just before reading the sample. A total of 10,000 events were acquired on BD LSRFortessa X20 (BD biosciences). Data were analyzed using FlowJo (Treestar ${ }^{\odot}$ V10).

\section{Histology and Immunohistochemistry}

$10^{5} \mathrm{HEKa}$ seeded in 24 -well culture plates containing sterile coverslips were incubated at $37^{\circ} \mathrm{C}$ until 100\% confluent. They were then co-cultured with LiSA for 1h, fixed and permeabilized with $1 \%$ triton-X for $15 \mathrm{~min}$ and stained with mouse anti-human IL-33 monoclonal antibody (Nessy-1 ALX-804-840PF, 1:200 dilution, Enzo Life Sciences, Exeter, UK) and rabbit anti-human cytokeratin 14 polyclonal antibody (PA5-1672, 1:200 dilution, Thermo Fisher Scientific). Cultures were incubated with secondary antibodies (biotinylated anti-mouse, 1:200 dilution, Vector 

IgG Alexa Fluor Plus 488 (Invitrogen). Images were acquired at a magnification of 20X using a fluorescent microscope (Olympus, BX51). Histological sections were prepared from snap frozen 185 healthy human skin or NC/Tnd mouse samples embedded in OCT (KP Cryo-Compound, CellPath 186 Services, UK). $10 \mu \mathrm{m}$ sections were stained with anti-human or anti-mouse CDSN, DSC-1 or DSG1 187 (1:200, Thermo Fisher Scientific, 1:1000 Santa Cruz respectively) and detected using anti-rabbit IgG Texas Red ${ }^{\mathrm{TM}}$ (Invitrogen) or Alexa Fluor ${ }^{\mathrm{TM}}$ 594-conjugated secondary antibodies. Images were captured at a magnification of $20 \mathrm{X}$ using an Eclipse Ci fluorescent microscope (Nikon, Surrey, UK) and a SPOT camera (Image solutions Inc, Preston, UK) or using Olympus BX51 camera.

\section{Densitometry analysis}

194 To quantitatively analyze expression levels, the mean gray intensity of five randomly-selected $1955 \mu \mathrm{m}$ square fields from immunofluorescent staining samples and protein bands from Western 196 blots were measured by using Image J software (Ver 1.53a, Wayne Rasband, National Institutes 197 of Health, Bethesda, MD, USA).

\section{Trans-well Assay}

200 Trans-well assays were performed in 24-well trans-well $(6.5 \mathrm{~mm}$ diameter, $0.4 \mu \mathrm{m}$ pore size, 201 Costar). Normal Human Epidermal Keratinocytes (NHEK) were added to the lower chamber and 202 live S. aureus (LiSA) was added to the upper chamber. After $6 \mathrm{~h}$ incubation at $37^{\circ} \mathrm{C}$, the supernatant was collected for analysis of type 2 cytokines by ELISA. 
Filtered supernatant from S. aureus (FSA) fractionated by size exclusion centrifugal filter columns

208 (Amicon Ultra 15, 100kDa or 50kDa cut-off membranes) and centrifuged for $15 \mathrm{~min}$ at $3,000 \mathrm{~g}$

209 ( $\left.4^{\circ} \mathrm{C}\right) .200 \mathrm{ml}$ of FSA was fractionated using 100kDa Amicon Ultra 15 as described above. Retained

210 fractions were then pooled and then fractionated further using Superose ${ }^{\circledR} 6$ Fast Protein Liquid

211 Chromatography (FPLC) column $(5-5000 \mathrm{kDa}) .48$ fractions of $0.5 \mathrm{ml}$ were collected and stored 212 at $-20^{\circ} \mathrm{C}$ until required.

Sodium Dodecyl Sulphate-Polyacrylamide Gel Electrophoresis

215 Sodium dodecyl sulphate-polyacrylamide gel electrophoresis (SDS-PAGE) was used to analyse 216 the molecular weights of FSA proteins using standard methodology. Gels were then stained with 217 Instant Blue Coomassie-based gel stain or silver stain using standard protocols.

Mass spectrometry

220 Mass spectrometry was used to identify proteins in the bioactive peak derived from the FPLC size 221 fractionation. In-gel digestion and tandem mass spectrometry analysis was performed by the 222 Protein Mass Spectrometry Core Facility, Faculty of Biology, Medicine and Health, University of 223 Manchester. The data was generated against known protein sequences in Swissprot TrEMBL 224 database using Firmicutes phylum (which includes S. aureus) as the most optimal taxonomic level 225 available in the database.

\section{Candidate gene cloning and expression}

228 Details of cloning and expression of candidate genes using the E. coli vector system are provided 229 in the Methods section of this article's Online Repository. 

non-silencing control siRNA (AllStars negative control, 1027281) were purchased from Qiagen. NHEK were seeded at a density of $1 \times 10^{4}$ one day prior to transfection. After $24 \mathrm{~h}$, cells were transfected with $5 \mathrm{~nm}$ siRNA using $2.5 \mu \mathrm{l}$ of TransIT-TKO transfection reagent (Mirus Bio, UK). Experiments were performed on transfected cells at least two days after siRNA treatments. Knockdown efficiency of TLR2 siRNA mediated gene expression was confirmed by Real-time PCR.

\section{Animals}

$241 \mathrm{NC} /$ Tnd mice were generated in the laboratory of comparative animal medicine in Tokyo 242 University of Agriculture and Technology. MSM/Ms mice were purchased from RIKEN 243 Bioresource as wild-type (WT) controls. All animals were kept in a clear acrylic cage and had free access to standard chow and water. They were kept in specific-pathogen free housing to prevent the natural development of eczema that occurs if these mice are housed in conventional housing. Temperature and humidity of the animal room were $22 \pm 4^{\circ} \mathrm{C}$ and $40 \pm 15 \%$, respectively. The animal room was maintained on a 12:12-hour light-dark cycle. All animal experiments complied with the guidelines of University Animal Care and Use Committee of the Tokyo University of Agriculture and Technology, as well as with the guidelines of Science Council of Japan for the use of laboratory animals.

Topical application of filtered supernatant from $S$. aureus, S. epidermidis or allergen extracts $100 \mu 1$ of filtered supernatant stimulated with $S$. aureus (FSA) $\left(10^{7} \mathrm{CFU} / \mathrm{ml}\right.$ low dose or $10^{8}$

$254 \mathrm{CFU} / \mathrm{ml}$ high does) or $S$. epidermidis (FSE, $10^{8} \mathrm{CFU} / \mathrm{ml}$ ) was applied to the back skin of the mice once daily for four weeks. According to the manufacturer's instruction, $100 \mathrm{mg}$ of DF ointment was applied to the back skin of the mice. Application was performed twice/week for 4 weeks. Skin barrier disruption was performed by $4 \%$ SDS before the application of FSA, FSE and DF. 
259 Clinical eczema severity and scratching behavior scoring in mice

260 Clinical eczema scores in mice were assessed as previously described. ${ }^{20}$ Briefly, the total clinical 261 severity score was defined in individual mice as the sum of the individual scores graded as 0 (none), 2621 (mild), 2 (moderate), 3 (severe) for each of five signs and symptoms (itch, erythema/hemorrhage, 263 edema, excoriation/erosion, and scaling/dryness).

Scratching frequency and duration was measured for 30min each week and analyzed

265

\section{Trans-epidermal water loss measurement in mice}

Trans-epidermal water loss (TEWL) was measured using Tewameter ${ }^{\circledR}$ TM300 from Courage + Khazaka electronic GmbH (Cologne, Germany), once weekly for four weeks. Temperature and humidity were maintained at $22 \pm 0.5^{\circ} \mathrm{C}$ and $50 \% \pm 10 \%$ respectively before the measurements. The measurements for each mouse were taken three times and the mean value was calculated.

\section{Immunoblotting}

Whole cell lysates were isolated from the back skin of the mice using radioimmunoprecipitation assay buffer. After SDS-PAGE using a 10\% gel, immunoblotting was performed with anti-mouse CDSN monoclonal antibody (1:500; Santa Cruz), anti-mouse DSG-1 monoclonal antibody

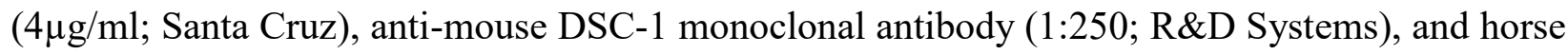
radish peroxidase-conjugated secondary antibody (1:1000; Cell Signaling). Positive reactions were visualised using Immobilon western chemiluminescent HRP substrate (Millipore). 
283 All in vitro and ex vivo experiments were carried out with a minimum of three technical and two284 three biological replicates as detailed in the figure legends. In vivo experiments were performed 285 twice with six mice per group. One-way ANOVA was used for single factor data with 3 or more 286 groups followed by comparisons to a control group using Dunnett's post-hoc test. However, if 287 normality could not be assumed using the QQ residual plot, statistical comparisons between groups 288 was determined using the Kruskal Wallis test with Dunn's multiple comparisons and adjusted P 289 values reported. Single factor data with less than three groups were analysed using the unpaired t290 test and the two-tailed P value reported. All analysis was performed using GraphPad Prism 8 291 Version 8.4.2 (GraphPad Software, Inc. CA, USA). A probability value of less than 0.05 was 292 considered statistically significant. Flow cytometry data were analysed using FlowJo (Treestar 293 V10) and represented as mean \pm standard error of the mean (SEM). 


\section{RESULTS}

\section{S. aureus, but no other staphylococcal species, induces type 2-promoting cytokines}

Although $S$. aureus is the major bacterial species associated with AD flares, ${ }^{21,22}$ the comparative ability of other staphylococcal species and skin flora to stimulate type 2-promoting cytokines by skin keratinocytes has not previously been directly evaluated. Of seven different staphylococcal species we investigated, only live $S$. aureus (LiSA) was able to trigger IL-33 and TSLP release by NHEK (Fig. 1a). S. epidermidis, the predominant bacterial skin commensal, was without any type2 immune potential. Furthermore, Group A streptococci (GAS), the other major bacterial skin pathogen, was also unable to stimulate release of these type 2- promoting cytokines (Fig. 1a). IL33 and TSLP release was not limited to one strain of $S$. aureus but could be demonstrated in six different strains and mutants tested, including RN4220, RN4220; $\Delta \operatorname{tagO}$, RN4220; $\Delta$ spa SH1000, SH1000; $\triangle$ FnBP (Fig. 1b). The unique proinflammatory effect of LiSA was dose-dependent (Fig. 1c) and detected after only $2 \mathrm{~h}$, in keeping with release of a prestored cytokine pool rather than de novo synthesis (Fig. 1d). To support this supposition, IL-33 demonstrated in the nuclei of unstimulated $\mathrm{HEKa},{ }^{23}$ dissipated after stimulation with live $S$. aureus (Fig. 1e). Live S. aureusinduced IL-33 release was associated with an increase in DAPI uptake and Annexin V expression, indicating an association with cell death (Fig. 1f-h).

Having shown that live S. aureus is unique in its ability to induce Th2-promoting cytokine release by human keratinocytes in vitro, we next investigated whether its bioactivity resided in the bacterial cell wall or secretome.

\section{S. aureus secretome induces Th2-promoting cytokines}

Heat-killed S. aureus (HKSA) was unable to induce cytokine release in primary human 317 keratinocytes, suggesting that the bioactivity did not reside in the cell wall of the bacteria (Fig. 1b). This was explored further by addition of the purified $S$. aureus bacterial wall components PGN and LTA to stimulate TLR2 signalling, as this has previously been reported to drive 
immunopathology. ${ }^{24}$ Neither exogenous PGN nor LTA induced IL-33 or TSLP release (Fig. 2a, b). Anti-TLR2 blocking antibodies (Fig. 2b) and siRNA knockdown of TLR2 in keratinocytes (Fig. 2c) did not suppress either IL-33 or TSLP release by FSA.

In contrast, experiments where LiSA and NHEK were separated by a $0.4 \mu \mathrm{m}$ pore membrane demonstrated that the type- 2 immune promoting bioactivity resided within the bacterial secretome (FSA) (Fig. 3a). The FSA bioactivity was heat-labile, but not affected by addition of antibiotics to remove any contaminating LiSA (Fig. 3b). Interestingly, in contrast to LiSA, coculture of FSA with NHEK did not induce either DAPI uptake or Annexin V expression, indicating that the bioactivity was independent of cell death (Fig. 3c-e).

\section{Skin barrier disruption and eczema induced by $S$. aureus secretome}

We then explored whether the secretome-derived factor was bioactive in ex vivo human skin organ culture and in an in vivo mouse eczema model. Using human skin organ explants from nonatopic adults undergoing abdominal skin excision for treatment of obesity, we demonstrated that sterile filtered supernatant from $S$. aureus (FSA), but not $S$. epidermidis (FSE), common house dust mite (DP \& DF) or pollen aeroallergens (Ragweed) stimulated release of both IL-33 and TSLP (Fig. 4awith $\mathrm{AD}$ is inherently different to that of non-atopic individuals. LiSA induced release of similar taken from children with moderate-to-severe AD and non-atopic controls, indicating that the Th2- 
(Fig. 4g-h). This suggested that exposure to S. aureus-derived bioactive factor(s) through a

347 disrupted skin barrier, rather than an inherent immune hyperactivity, is the important driver of 348 disease. $^{26}$

The NC/Tnd Japanese fancy bred mouse is an excellent animal model of human AD, in that pups from this inbred strain in conventional, but not in specific-pathogen free housing, develop eczema soon after weaning at $6-8$-weeks old. The immunohistochemistry mirrors that of human $352 \mathrm{AD}$ and the disease responds to topical corticosteroids and tacrolimus ointment. ${ }^{27,28}$ Eczema in this mouse model is aggravated by $S$. aureus as topical antibiotics suppress clinical disease and Th2 response. ${ }^{29}$ Using this mouse model in specific pathogen free housing, we demonstrate that daily application of FSA and house dust mite allergen (DF), but not FSE for four weeks to SDS primed skin induces a dose-dependent flare in clinical eczema (Fig. 5a-b) associated with increased scratching behaviour (Fig. 5c) and disruption in skin barrier function measured by TEWL (Fig. $5 d)$.

Associated with the clinical eczema flare, skin histology showed marked hyperkeratosis of the epidermis where FSA (or house dust mite allergen) had been applied (Fig. 6a). As with the human explant model, expression of corneodesmosome proteins (particularly corneodesmosin) in the stratum corneum on immunofluorescence appeared to be disrupted (Fig. 6a). Densitometry showed that FSA reduced intensity of expression of CDSN, DSC-1 and CSG-1 compared with the control, with more variable reduction after application of DF and FSE (Fig. 6b-d). Western blot analysis and densitometry from mouse skin illustrated that CDSN was significantly degraded by FSA and DF compared with the control and FSE (Fig. 6e, 6f). There was evidence of degradation of DSC-1 by FSA, DF and FSE (Fig. 6e, 6g), but DF and not FSA degraded DSG-1 (Fig 6e, 6h). In addition to the local cutaneous effects, application of FSA to the skin of these NC/Tnd mice led to significant increases in plasma concentrations of TSLP (Fig. 6i) and IL-33 (Fig 6j). This is in 370 keeping with the known association between AD, circulating IL-33 and TSLP and other noncutaneous allergies such as food allergies and allergic asthma as part of the "atopic march". ${ }^{30}$ 
372 Overall, the in vivo mouse data highlight the Th2-promoting and skin barrier disruptive effects of the secretome derived from $S$. aureus.

The critical role of IL-33 in inducing both the clinical disease and TEWL was then explored by treating the NC/Tnd mice with a neutralizing anti-IL-33 monoclonal antibody at the time of the first application of FSA. FSA-treated mice, including those given an injection of the isotype antibody developed clinical evidence of eczema and increased TEWL, while administration of the anti-IL-33 monoclonal antibody completely abrogated the disease (Fig. 7a-d). We conclude that, at least in this mouse model, IL-33 is essential for the development of $S$. aureus-induced eczema. This is in keeping with the results of recent phase $2 \mathrm{~A}$ study of 12 adults with moderate to severe AD, which showed rapid improvement in Eczema Area and Severity Index 50 after a single injection of etokimab (an IgG1 anti-IL-33 monoclonal antibody). ${ }^{31}$

\section{Sbi is the $S$. aureus $T h 2$-promoting factor}

Having demonstrated the unique Th2-promoting activity of the $S$. aureus secretome on both human and mouse skin in vitro and in vivo, as well as the essential role of IL-33 in the pathogenesis of eczema in the $\mathrm{NC} / \mathrm{Tnd}$ mouse model, we proceeded to purify and characterize the specific bioactive factor. The bioactivity of FSA was retained by both a $50 \mathrm{kDa}$ and a $100 \mathrm{kDa}$ molecular weight cutoff filter (data not shown). We used a Superose ${ }^{\circledR} 6$ size exclusion chromatography column with a separation range of $5-5,000 \mathrm{kDa}$ to further fractionate the FSA proteins retained by the $100 \mathrm{kDa}$ column (Fig. 8a).

Mass spectroscopy of the most bioactive fraction revealed three potential S. aureus specific proteins (Fig. 8b). These proteins (DihydroLipoyl Dehydrogenase (DLD), Second Immunoglobulin-Binding Protein (Sbi), and Pyruvate Dehydrogenase Alpha-subunit (PDHa)) were expressed in an E. coli system and the purified products screened for Th2-promoting cytokine release by NHEK. Although not identified by mass spectroscopy, SplD, recently considered potentially important in a $S$. aureus-induced asthma mouse model, ${ }^{32}$ was also expressed in the $E$. 
coli expression system, the protein purified, and its activity assessed. The Th2-promoting 399 bioactivity of a commercially available $S$. aureus protease, Leucine Aminopeptidase 3 (LAP-3), 400 was also tested. Sbi was the only protein to show activity in the NHEK assays comparable with 401 FSA (Fig. 8c). DLD, PDHa, LAP-3 and SplD showed no activity above baseline (Fig 8c). The 402 reduced IL-33 and TSLP release after stimulation with FPLC-purified FSA Sbi fraction is in 403 consistent with loss of protein during the purification process (relative abundance of Sbi in the 404 FPLC-purified fraction compared with the FSA starting material was 2 versus 8 units as assessed 405 using mass spectroscopy). To provide additional evidence that Sbi is indeed the S. aureus bioactive 406 factor, E. coli pre- and post-transfection with the S. aureus sbi gene were added to NHEK. Sbi407 expressing E. coli induced IL-33 release, while the non-transfected E. coli did not (Fig. 8d). Finally, 408 we showed that two loss of function $\mathrm{Sbi}^{-}$S. aureus mutants derived from the Newman S. aureus 409 strain (Sbi ${ }^{-}$and $\mathrm{pRMC} 2: \mathrm{Sbi}^{-}$mutants), the latter added in tetracycline-free medium to ensure non410 expression of the Sbi protein) induced little or no IL-33 or TSLP release by NHEK, either using 411 co-culture of the live bacteria when compared with controls including a Spa mutant from the same 412 strain (Fig. 8e), or filtered supernatants (Fig. 8f). Sbi is present in reference proteomes USA300 413 (methicillin resistant), ${ }^{33}$ Newman and NCTC $8325,{ }^{34}$ suggesting that it is a conserved protein 414 within $S$. aureus species. 
416 We have identified the Second Immunoglobulin-Binding Protein as a key Th2-promoting bioactive

417 factor of $S$. aureus, responsible for its dominant role as a trigger of cutaneous atopic disease in 418 humans and mouse. Secretome from $\mathrm{Sbi}^{-}$deficient $S$. aureus mutants induced little or no type 2 419 immune activity. Co-culture of NHEK with live Sbi- mutant bacteria resulted in significantly less 420 but detectable IL-33 activity, probably due to nonspecific cytotoxic effects particularly with more 421 prolonged culture. Until now the precise role of $S$. aureus in AD type 2 driven immunopathology 422 has been poorly understood. Using human explants and a mouse model of AD, we demonstrate the 423 critical link between the $S$. aureus secretome and IL-33 release in mediating eczema, in keeping 424 with the effectiveness of an anti-IL-33 monoclonal antibody in a recent phase $2 \mathrm{~B}$ clinical trial. ${ }^{33}$ 425 We also show that the $S$. aureus secretome degrades CDSN. CDSN is a key non-covalently bound 426 component of the stratum corneum corneodesmosome, functioning to maintain skin barrier 427 integrity. ${ }^{35}$ Disruption of the skin barrier allows for ingress of microbes and allergens into the 428 epidermis leading to initiation of the type 2 host immune response. This is supported by the comparative ability of both human AD-derived and healthy keratinocytes to release IL-33 in response to the $S$. aureus secretome.

Importantly, neither the predominant skin commensal $S$. epidermidis, nor another skin pathogen Group A streptococcus contains Sbi homologs in their genome. Using Basic Local 433 Alignment Search Tools (BLAST, UniProt.org and NCBI), Sbi was found to be unique to 434 Staphylococcus aureus amongst the Bacilli genus and 100\% homologous across key USA300 435 (methicillin resistant), Newman, and NCTC 8325 strains. Homology across other recorded $S$. 436 aureus strains (Uniprot) ranges from 91.7-99.8\%. Sbi is known to be present in 16 clinical S. aureus 437 strains ${ }^{36}$ supporting our conclusion that the bioactivity is not unique to one clinical isolate. Finally, we have excluded other virulence factor candidates such as SplD as contributing to this bioactivity 
439 in the skin, unlike its possible role in the lung, demonstrating the importance of considering the infection niche and responding immune cells. ${ }^{37}$

Sbi was first discovered in 1998 and is a 436-amino acid protein with four globular domains, two of which are homologous to Protein A, allowing binding to the Fe domain of IgG, thus inhibiting neutrophil-mediated phagocytosis. ${ }^{36,38}$ The other two domains interfere with complement activity. ${ }^{39}$ Unlike the "first" immunoglobulin binding protein (Protein A), Sbi contains a signal peptide and is present in the secretome. ${ }^{19}$ The Immunoglobulin binding domains of Sbi have previously been shown to interact with Ig domains of the TNFR1 receptors on murine macrophages in vitro and in vivo to induce IL- 6 and TNF- $\alpha .{ }^{40}$ TNFR1 is expressed on human keratinocytes and $S$. aureus can increase its expression further. ${ }^{41,42}$ However, the effect of Sbi on the skin and Th2-promoting cytokines has not previously been examined. Immunoglobulin-like domains are common to several different receptor types found on keratinocytes including members of the IL-1R superfamily. It is therefore possible that Sbi binds to a receptor on the surface of keratinocytes, through recognition of an Ig domain, inducing TSLP and IL-33 release. Alternatively, Sbi has been shown to induce epidermal growth factor receptor (EGFR) in macrophages in vitro and in vivo. ${ }^{43}$ As activation of EGFR is known to be involved in the induction of IL-33 and TSLP transcription in keratinocytes, ${ }^{43,44} \mathrm{Sbi}$ induction of EGFR may be an alternative pathway for the release of IL-33 and TSLP from NHEK. Further studies are required to elucidate the exact mechanism of action of Sbi based on these possibilities.

Regarding the ability of FSA to degrade and disrupt CDSN expression as show in the immunohistology and Western blots, this is likely to be indirect effect of Sbi, as this factor contains no inherent protease activity. Endogenous keratinocyte-derived kallikreins are important mediators of skin barrier disfunction, as exemplified in Netherton syndrome. ${ }^{45}$ We have previously shown that kallikrein- 5 can be activated by changes in skin $\mathrm{pH}$ in $\mathrm{NC} / \mathrm{Tn}$ mice leading to disrupted skin 
barrier function. ${ }^{20}$ The complex interplay and exact mechanism by which Sbi might active these endogenous kallikreins to induce skin barrier dysfunction requires further investigation.

Over the last decade there have been huge advances in our understanding of host pathogen interactions, establishing a link between humans and microbial diversity in health-related outcomes. ${ }^{46}$ The prime aim of this study was to address the question as to what makes $S$. aureus the undisputed master of its bacterial class in promoting atopy. Sbi is an important piece of the puzzle. In addition, this is the first time a pathogen-specific molecule has been identified to induce rapid release of IL-33, independently of cell death and TLR2 recognition. Previous studies have suggested a possible role of S. aureus TLR2 ligands in the induction of type 2 responses in mouse skin and human keratinocytes. In the study by Brauweiler et al,${ }^{47}$ lipoteichoic acid injected intradermally into mouse skin led to an increase in TSLP mRNA and to a lesser extent IL-33, but protein levels were not assessed. Furthermore, it is not possible to conclude if the ligand had a direct effect on keratinocytes or acted indirectly through other resident skin immune cells. Additionally, the relevance of this murine model to human skin is unclear. Vu et al ${ }^{48}$ found that a synthetic diacylated lipoprotein but not PGN or $\mathrm{Pam}_{3} \mathrm{CSK}_{4}$ induced small amounts of TSLP in human keratinocytes but the authors did not assay IL-33. SplD induced IL-33 release from airway epithelial cells type II within an hour. ${ }^{32}$ The lack of IL-33 release by skin NHEK after addition of SplD in our study suggests that molecular triggers of atopy, including S. aureus associated virulence factors, can be tissue-specific.

In summary, we believe that our novel results significantly advance the understanding of the etiology of AD, providing compelling evidence that a S. aureus protein drives IL-33 release in skin, triggering the allergic-type phenotype associated with $\mathrm{AD}$. Further research is now required to determine the exact mechanism by which Sbi induces IL-33 release, and also how it degrades corneodesmosome proteins critical to skin barrier function. 
Acknowledgements The authors would like to thank Dr Gianni Mistrello for generous provision

488 of house dust mite and ragweed allergens, and Professor Joan Geoghegan, Trinity College

489 Dublin, Professor Andrew McBain and Dr Gouxing Xia for Staphylococcal species and S. aureus

490 strains. Also, The Genome Editing Unit at The University of Manchester, Carl Bradford,

491 Professor Ian Roberts, Miss Marie Goldrick and Ms Elizabeth Lord for valuable technical

492 assistance and advice with cloning, expression and purification, SplD cloning, expression and

493 purification respectively, as well as Ms Ryo Muko, Tokyo University of Agriculture \&

494 Technology, Tokyo, Japan for help with densitometry measurements.

495

496 Author contributions The impetus and direction of this study were initiated by PDA and JLP, 497 who oversaw and coordinated the experiments in their entirety. AT and HM oversaw and 498 coordinated all mouse experiments. CO’N, along with PDA and JLP supervised the work of AAK.

499 AAL, HW, AMA, SYT, CS and YA conducted the in vitro experiments. HW and CS conducted 500 the ex vivo skin explant experiments. KM conducted all in vivo mouse experiments. HB was 501 instrumental and provided technical advice and support for the E. coli cloning experiments. PDA 502 wrote the first draft of the manuscript, which then had input from all other co-authors, who 503 reviewed drafts and the final version of this manuscript. 
505 1. Poulakou G, Lagou S, Tsiodras S. What's new in the epidemiology of skin and soft tissue 506 infections in 2018? Curr Opin Infect Dis. 2019;32:77-86.

507 2. Lunjani N, Hlela C, O'Mahony L. Microbiome and skin biology. Curr Opin Allergy Clin 508 Immunol. 2019;19:328-333.

3. Esposito S, Noviello S, Leone S. Epidemiology and microbiology of skin and soft tissue infections. Curr Opin Infect Dis 2016;29:109-15.

4. Kim J, Kim BE, Ahn K, Leung DYM. Interactions between atopic dermatitis and Staphylococcus aureus infection: Clinical implications. Allergy Asthma Immunol Res 2019;11:593-603.

5. Paller AS, Kong HH, Seed P, Naik S, Scharschmidt TC, Gallo RL, et al. The microbiome in patients with atopic dermatitis. J Allergy Clin Immunol 2019;143:26-35.

6. Weidinger S, Novak N. Atopic dermatitis. Lancet 2016;387:1109-1122.

7. Lacey KA, Geoghegan JA, McLoughlin RM. The role of Staphylococcus aureus virulence factors in skin infection and their potential as vaccine antigens. Pathogens 2016;5:(1).

8. Seiti Yamada Yoshikawa F, Feitosa de Lima J, Notomi Sato M, Álefe Leuzzi Ramos Y, Aoki V, Leao Orfali R. Exploring the Role of Staphylococcus Aureus Toxins in Atopic Dermatitis.

9. Al Kindi A, Alkahtani AM, Nalubega M, El-Chami C, O'Neill C, Arkwright PD, et al. Staphylococcus aureus internalized by skin keratinocytes evade antibiotic killing. Front Microbiol 2019;10:2242.

10. Askarian F, Wagner T, Johannessen M, Nizel V. Staphylococcus aureus modulation of innate immune responses through Toll-like (TLR), (NOD)-like and C-type lectin (CLR) receptors. FEMS Microbiol Rev 2018;42: 656-671. 
11. Leung DYM, Berdyshev E, Goleva E. Cutaneous Barrier Dysfunction in Allergic Diseases. J Allergy Clin Immunol. 2020;145:1485-1497.

12. Smith FJ, Irvine AD, Terron-Kwiatkowski A, Sandilands A, Campbell LE, Zhao Y, et al. Loss-of-function mutations in the gene encoding filaggrin cause ichthyosis vulgaris. Nat Genet. 2006;38:337-42.

13. Honda T, Kabashima K. Reconciling innate and acquired immunity in atopic dermatitis. J Allergy Clin Immunol. 2020;Feb 25.

14. Simpson EL, Bieber T, Guttman-Yassky E, Beck LA, Blauvelt A, Cork MJ, et al. Two Phase 3 Trials of Dupilumab versus Placebo in Atopic Dermatitis. N Engl J Med. 2016;375:23352348.

15. Geoghegan JA, Irvine AD, Foster TJ. Staphylococcus aureus and Atopic Dermatitis: A Complex and Evolving Relationship. Trends Microbiol 2018;26:484-497.

16. Nakamura Y, Oscherwitz J, Cease KB, Chan SM, Muñoz-Planillo R, Hasegawa M, et al Staphylococcus $\delta$-Toxin Induces Allergic Skin Disease by Activating Mast Cells. Nature 2013;503:397-401.

17. Casanova JL, Abel L. Human genetics of infectious diseases: Unique insights into immunological redundancy. Semin Immunol 2018;36:1-12.

18. Fischer A, Rausell A. What do primary immunodeficiencies tell us about the essentiality/redundancy of immune responses? Semin Immunol 2018;36:13-16.

19. Smith EJ, Visai L, Kerrigan SW, Speziale P, Foster TJ. The Sbi protein is a multifunctional immune evasion factor of Staphylococcus aureus. Infect Immun 2011;79:3801-3809.

20. Jang H, Matsuda A, Jung K, Karasawa K, Matsuda K, Oida K, et al. Skin pH is the master switch of kallikrein 5-mediated skin barrier destruction in a murine atopic dermatitis model. J. Invest. Dermatol. 2016;136:127-135. 
21. Byrd AL, Deming C, Cassidy SKB, Harrison OJ, Ng WI, Conlan S, et al. Staphylococcus aureus and Staphylococcus epidermidis strain diversity underlying pediatric atopic dermatitis. Sci Transl Med 2017;9:(397).

22. Fyhrquist, N., Muirhead G, Prast-Nielsen S, Jeanmougin M, Olah P, Skoog T, et al. Microbehost interplay in atopic dermatitis and psoriasis. Nat Commun 2019;10:4703.

23. Moussion C, Ortega N, Girard JP. The IL-1-like cytokine IL-33 is constitutively expressed in the nucleus of endothelial cells and epithelial cells in vivo: a novel 'alarmin'? PLoS One 2008;3:e3331.

24. Lan F, Zhang N, Holtappels G, De Ruyck N, Krysko O, Van Crombruggen K, et al. Staphylococcus aureus induces a mucosal type 2 immune response via epithelial cell-derived cytokines. Am J Respir Crit Care Med 2018;198:452-463.

25. Jonca N, Guerrin M, Hadjiolova K, Caubet C, Gallinaro H, Simon M, et al. Corneodesmosin, a component of epidermal corneocyte desmosomes, displays homophilic adhesive properties. J Biol Chem 2002;277:5024-5029.

26. Palmer CN, Irvine AD, Terron-Kwiatkowski A, Zhao Y, Liao H, Lee SP, et al. Common lossof-function variants of the epidermal barrier protein filaggrin are a major predisposing factor for atopic dermatitis. Nat Genet 2006;38:441-446.

27. Noguchi A, Tominaga M, Takahashi N, Matsuda H, Kamata Y, Umehara Y, et al. Differences in therapeutic effects of topically applied corticosteroid and tacrolimus on atopic dermatitislike symptoms in NC/Nga mice. J Dermatol Sci 2017;86:54-62.

28. Amagai Y, Matsuda H, Tanaka A. Abnormalities in itch sensation and skin barrier function in atopic NC/Tnd mice. Biol Pharm Bull 2013;36:1248-1252.

29. Matsui K, Nojima Y, Kajiwara Y, Busujima K, Mori Y. Topical application of doxycycline inhibits Th2 cell development mediated by Langerhans cells and exerts a therapeutic effect on atopic dermatitis. J Pharm Pharm Sci 2020;23:86-99. 
30. Lowe AJ, Leung DYM, Tang MLK, Su JC, Allen KJ. The skin as a target for prevention of the atopic march. Ann Allergy Asthma Immunol 2018;120:145-151.

31. Chen YL, Gutowska-Owsiak D, Hardman CS, Westmoreland M, MacKenzie T, Cifuentes L, et al. Proof-of-concept clinical trial of etokimab shows a key role for IL-33 in atopic dermatitis pathogenesis. Sci Transl Med 2019;11:(515).

32. Teufelberger AR, Nordengrün M, Braun H, Maes T, De Grove K, Holtappels G, et al. The IL-33/ST2 axis is crucial in type 2 airway responses induced by Staphylococcus aureusderived serine protease-like protein D. J Allergy Clin Immunol 2018;141:549-559.e7.

33. Diep BA, Gill SR, Chang RF, Phan TH, Chen JH, Davidson MG, et al. Complete genome sequence of USA300, an epidemic clone of community-acquired methicillin-resistant Staphylococcus aureus. Lancet 2006;367:731-739.

34. Berscheid A, Sass P, Weber-Lassalle K, Cheung AL, Bierbaum G. Revisiting the genomes of the Staphylococcus aureus strains NCTC 8325 and RN4220. Int J Med Microbiol 2012;302:84-87.

35. Jonca N, Caubet C, Guerrin M, Simon M, Serre G. Corenodesmosin: Structure, function and involvement in pathophysiology. Open Dermatol J 2010;4:36-45.

36. Smith EJ, Visai L, Kerrigan SW, Speziale P, Foster TJ. The Sbi protein is a multifunctional immune evasion factor of Staphylococcus aureus. Infect Immun 2011;79:3801-3809.

37. Stentzel S, Teufelberger A, Nordengrün M, Kolata J, Schmidt F, van Crombruggen K, et al. Staphylococcal serine protease-like proteins are pacemakers of allergic airway reactions to Staphylococcus aureus. J Allergy Clin Immunol 2017;139:492-500.

38. Zhang L, Jacobsson K, Vasi J, Lindberg M, Frykberg L. A second IgG-binding protein in Staphylococcus aureus. Microbiology 1998;144:985-91.

39. Koch TK, Reuter M, Barthel D, Böhm S, van den Elsen J, Kraiczy P, et al. Staphylococcus aureus proteins Sbi and Efb recruit human plasmin to degrade complement C3 and C3b. PLoS One 2012;7:e47638. 
603

604

605

606

607

608

609

610

611

612

613

614

615

616

617

618

619

620

621

622

623

624

625

626

40. Gonzalez CD, Ledo C, Giai C, Garófalo A, Gómez MI. The Sbi Protein Contributes to Staphylococcus aureus Inflammatory Response during Systemic Infection. PLoS One 2015;10:e0131879.

41. Wang X, Cheng D, Hu G, Liang L, Tan F, Xiao T, et al. Tumor Necrosis Factor (TNF) Receptor Expression Determines Keratinocyte Fate upon Stimulation with TNF-Like Weak Inducer of Apoptosis. Mediators Inflamm 2019;2019:2945083.

42. Aufiero B, Guo M, Young C, Duanmu Z, Talwar H, Lee HK, et al. Staphylococcus Aureus Induces the Expression of Tumor Necrosis Factor-Alpha in Primary Human Keratinocytes. Int J Dermatol 2007;46:687-94.

43. Meephansan J, Komine M, Tsuda H, Karakawa M, Tominaga S, Ohtsuki M. Expression of IL33 in the epidermis: The mechanism of induction by IL-17. J Dermatol Sci 2013;71:107-114.

44. Segawa R, Shigeeda K, Hatayama T, Dong J, Mizuno N, Moriya T, et al. EGFR transactivation is involved in TNF- $\alpha$-induced expression of thymic stromal lymphopoietin in human keratinocyte cell line. J Dermatol Sci 2018;89:290-298.

45. Kishibe M. Physiological and pathological roles of kallikrein-related peptidases in the epidermis. J Dermatol Sci 2019;95:50-55.

46. Integrative HMP (iHMP) Research Network Consortium. The Integrative Human Microbiome Project. Nature 2019;569:641-648.

47. Bauweiler AM, Goleva E, Leung DYM. Staphylococcus aureus lipoteichoic acid initiates a TSLP-basophil-IL-4 axis in the skin. J Invest Dermatol 2020;140:915-917.

48. Vu AT, Baba T, Chen X, Le TA, Kinoshita H, Xie Y, et al. Staphylococcus aureus membrane and diacylated lipopeptide induce thymic stromal lymphopoietin in keratinocytes through the toll-like receptor 2-toll-like receptor 6 pathway. J Allergy Clin Immunol 2010;126:985-93, 993.e1-3. 
Figure $1 S$. aureus induces IL-33 and TSLP release by human keratinocytes in vitro. a. Live

S. aureus (LiSA) $\left(10^{7} \mathrm{CFU} / \mathrm{ml}\right)$, but not other staphylococcus species, or Group A streptococci (GAS) induced IL-33 and TSLP by NHEK. S. cap: Staphylococcus capitis; S. car: Staphylococcus carnosus; S. coh: Staphylococcus cohnii; S. hem: Staphylococcus hemolyticus; S. len: staphylococcus lentis. b. All S. aureus strains tested induced IL-33 and TSLP release from NHEK. Cytokine release was c. dose- and d. time-dependent. e. IL-33 was constitutively expressed in nuclei of HEKa (top panel) and released after $1 \mathrm{~h}$ stimulation with LiSA (bottom panel). Cells stained with anti-human IL-33 monoclonal antibody (red) and anti-human cytokeratin 14 (green).

f. Dot plots of viable (Annexin $\mathrm{V}^{-} / \mathrm{DAPI}^{-}$), early apoptotic (Annexin $\mathrm{V}^{+} \mathrm{DAPI}^{-}$), late apoptotic $\left(\right.$ Annexin $\mathrm{V}^{+} / \mathrm{DAPI}^{+}$) and necrotic (Annexin $\mathrm{V}^{-} / \mathrm{DAPI}^{+}$) cells following stimulation with LiSA (10 CFU/ml) for up to 6 h. g. $\&$ h. LiSA cytotoxic activity after 6 h stimulation. Data represents three independent experiments performed in triplicate. Images taken at $20 \mathrm{X}$ magnification. $* P<0.01$, ** $P<0.001$ compared with the control. $P$-values determined by one-way ANOVA with Dunnett's multiple comparisons. Mean \pm standard error of the mean.

Figure $2 S$. aureus cell wall components have no bioactivity and its secretome induces IL-33 and TSLP release independent of TLR2. a. Exogenous bacterial peptidoglycan (PGN) 0$100 \mu \mathrm{g} / \mathrm{ml}$ and lipoteichoic acid (LTA) $0-100 \mu \mathrm{g} / \mathrm{ml}$ and did not induce release of IL-33 or TSLP by NHEK at $6 \mathrm{~h}$. b. Anti-TLR2 antibodies $(1$ or $10 \mu \mathrm{g} / \mathrm{ml})$ did not inhibit IL-33 or TSLP release induced by LiSA. c. Inhibiting TLR2 expression with $20 \mathrm{mM}$ of specific siRNA did not suppress FSAinduced release of IL-33 or TSLP by NHEKs at $6 \mathrm{~h}$. Scr $=$ scrambled siRNA control. Data represents three independent experiments performed in triplicate. ${ }^{*} P<0.001$ compared with the control. $P$-values determined by one-way ANOVA with Dunnett's multiple comparisons. Mean \pm standard error of the mean. 
Figure 3 Type 2-promoting bioactivity of $S$. aureus resides in the secretome. a. IL-33 release can be induced by NHEK when separated from the LiSA by a $0.4 \mu \mathrm{m}$ transmembrane filter. $\mathbf{b}$.

Bioactivity of FSA is destroyed by heating to $95^{\circ} \mathrm{C}$, but by treatment with penicillin/streptomycin (P/S). Cytokine release (IL-33 and TSLP) was measured by ELISA. c. Dot plots of viable (Annexin $\mathrm{V}^{-} / \mathrm{DAPI}^{-}$), early apoptotic $\left(\right.$Annexin $\left.\mathrm{V}^{+} \mathrm{DAPI}^{-}\right)$, late apoptotic (Annexin $\mathrm{V}^{+} / \mathrm{DAPI}^{+}$) and necrotic (Annexin $\mathrm{V}^{-} / \mathrm{DAPI}^{+}$) cells following stimulation with FSA for up to $6 \mathrm{~h}$. d. \& e. Quantification of FSA cytotoxic activity determined by $\mathrm{DAPI}^{+}$and Annexin $\mathrm{V}^{+}$staining of cells. All data are representative of three independent experiments performed in triplicate. $* P<0.05$, ** $P<0.001$ compared with the control. $P$-values were determined by one-way ANOVA with Dunnett's multiple comparisons. Mean \pm standard error of the mean.

\section{Figure 4 S. aureus Filtered Supernatant (FSA) induces TSLP and IL-33 and disrupts} corneodesmosome expression in human skin organ culture. a. \& b. FSA but not filtered supernatant from S. epidermidis (FSE), house dust mite allergens (D. pteronyssinus (DP), D. farinae (DF) or Ragweed allergen induces TSLP and IL-33 after $6 \mathrm{~h}$ and $12 \mathrm{~h}$. Three independent experiments performed in duplicate. ${ }^{*} P<0.05$, $* * P<0.001$ compared with the control. Mean \pm standard error of the mean. c. Corneodesmosin (CDSN), Desmocollin-1 (DSC1) and Desmoglein-1 (DSG-1) staining following 6h stimulation with FSA, FSE, and common aeroallergens DP, DF and ragweed. CDSN staining is disrupted following FSA treatment. Images are representative of three independent experiments performed in duplicate. Scale bars in $\mathrm{c}=$ $40 \mu \mathrm{m}$. df. Densitometric analysis for CDSN, DSC-1 and DSG-1. Mean of three independent experiments performed in duplicate. Bar represents median and * represents significant difference compared with control. g. \& h. Primary keratinocytes from 11 children with AD and 11 healthy controls released IL-33 and TSLP in response to LiSA but not heat-killed S. aureus 
677 (HKSA) or lipopolysaccharide (LPS). Data represents three independent experiments performed 678 in duplicate. ${ }^{* *} P<0.001$ compared with the control. Bar represents median.

Figure 5 S. aureus Filtered Supernatant (FSA) induces eczema and skin barrier disruption in the $\mathrm{NC} /$ Tnd mouse in vivo. $80-100 \mu$ l of low $\left(10^{7} \mathrm{CFU} / \mathrm{ml}\right)$ and high concentration $\left(10^{8}\right.$ CFU/ml) FSA, FSE, or $D$. farinae (DF) with concomitant 4\% SDS were applied topically to the backs of 8-week old NC/Tnd mice each day for four weeks. Mice were housed in specific pathogen free conditions. a. Representative images of NC/Tnd mice after application of 4\% SDS, or 4\% SDS and FSE, DF or FSA had been applied to the back daily for four weeks. b. Clinical eczema scores. c. Scratching behaviour and d. Trans-Epidermal Water Loss (TEWL). All data show results of six individual mice/group. ${ }^{* *} P<0.01$. Mean \pm standard error of the mean.

Figure 6 Filtered supernatant from $S$. aureus but not $S$. epidermidis increases plasma IL-33 and TSLP, particularly in NC/Tnd mice and disrupts epidermal corneodesomosme expression. a. Histological sections from eczema-prone NC/Tnd mice. 4\% SDS, or 4\% SDS and DF, FSE or FSA had been applied daily for four weeks. Images are representative of six individual mice/group performed in duplicate. b-d. Densitometric analysis. ${ }^{*} P<0.01, * * P<0.0001$ compared with the SDS control. Bar represents median. e. Western blots of CDSN, DSC-1 and DSG-1 native protein and degraded fragments from NC/Tnd mouse skin. Densitometric analysis of Western blots for CDSN (f) DSC-1 (g) and DSG-1 (h). Data representative of six individual mice/group. $* P<0.05, * * P<0.001$ compared with the naïve control. i \& j. Plasma IL-33 and TSLP concentrations in NC/Tnd and MSM/Ms. SDS: sodium dodecyl sulphate, FSE: filtered supernatant

699 from S. epidermidis, DF: D. farinae, FSA: filtered supernatant from S. aureus. Data are representative of six mice/group. ${ }^{* *} P<0.001$. Mean \pm standard error of the mean. 
Figure 7 FSA-induced eczema in the NC/Tnd mouse can be completely abrogated by neutralizing anti-IL-33 antibodies. $80-100 \mu \mathrm{l}$ of high $\left(10^{8} \mathrm{CFU} / \mathrm{ml}\right)$ concentration FSA with concomitant 4\% SDS were applied topically to the backs of 8 -week old NC/Tnd mice each day for four weeks. One group also received an intraperitoneal $10 \mu \mathrm{g}$ injection of anti-IL-33 monoclonal antibody with the first application of FSA, while a second group received an injection of an isotype control antibody. a. Representative images of NC/Tnd mice after application of 4\% SDS and FSA, FSA daily for four weeks, with or without isotype or anti-IL-33 mAb. b. Clinical eczema scores, c. scratching frequency and d. Trans-Epidermal Water Loss (TEWL) of naïve mice, and mice treated with 4\% SDS and FSA, isotype control or anti-IL-33 antibody. All data show results of six individual mice/group. ${ }^{* *} P<0.01$ compared with pre-treatment or the naïve group. Mean \pm standard error of mean.

Figure 8 Fractionation of $S$. aureus secretome (FSA) Th2-promoting activity by FPLC and identification of the active factor as $\boldsymbol{S}$. aureus Sbi. a. Bioactivity of FSA fractionated using Superose $^{\circledR} 6$ FPLC of $100 \mathrm{kDa}$ retention fraction (insert shows absorbance (280nm); main panel shows IL-33 release by NHEK after addition of specific FPLC fractions). b. S. aureus proteins identified from bioactive Superose ${ }^{\circledR} 6$ FPLC fractions by mass spectroscopy. c. IL-33 and TSLP release by NHEK after addition of E. coli vector-derived DLD, Sbi, PDHa, SplD, or exogenous LAP-3 for 6 h. d. E. coli expressing Sbi, but not untransfected E. coli induced IL-33 release by NHEK. e. LiSA, and f. FSA from Sbi- and pRMC2:Sbi-deficient strains induce less IL-33 and TSLP than parental Newton wild-type $S$. aureus. e. Spa-deficient LiSA induces the same IL-33 and TSLP as the Newton wide-type LiSA. All data are representative of two to three independent experiments performed in duplicate or triplicate. ${ }^{* *} P<0.0001$ compared with control. Mean \pm standard error of mean. DLD: dihydrolipoyl dehydrogenase, LiSA: live S. aureus, Sbi: Second immunoglobulin-binding protein, and PDHa: pyruvate dehydrogenase alpha-subunit. 

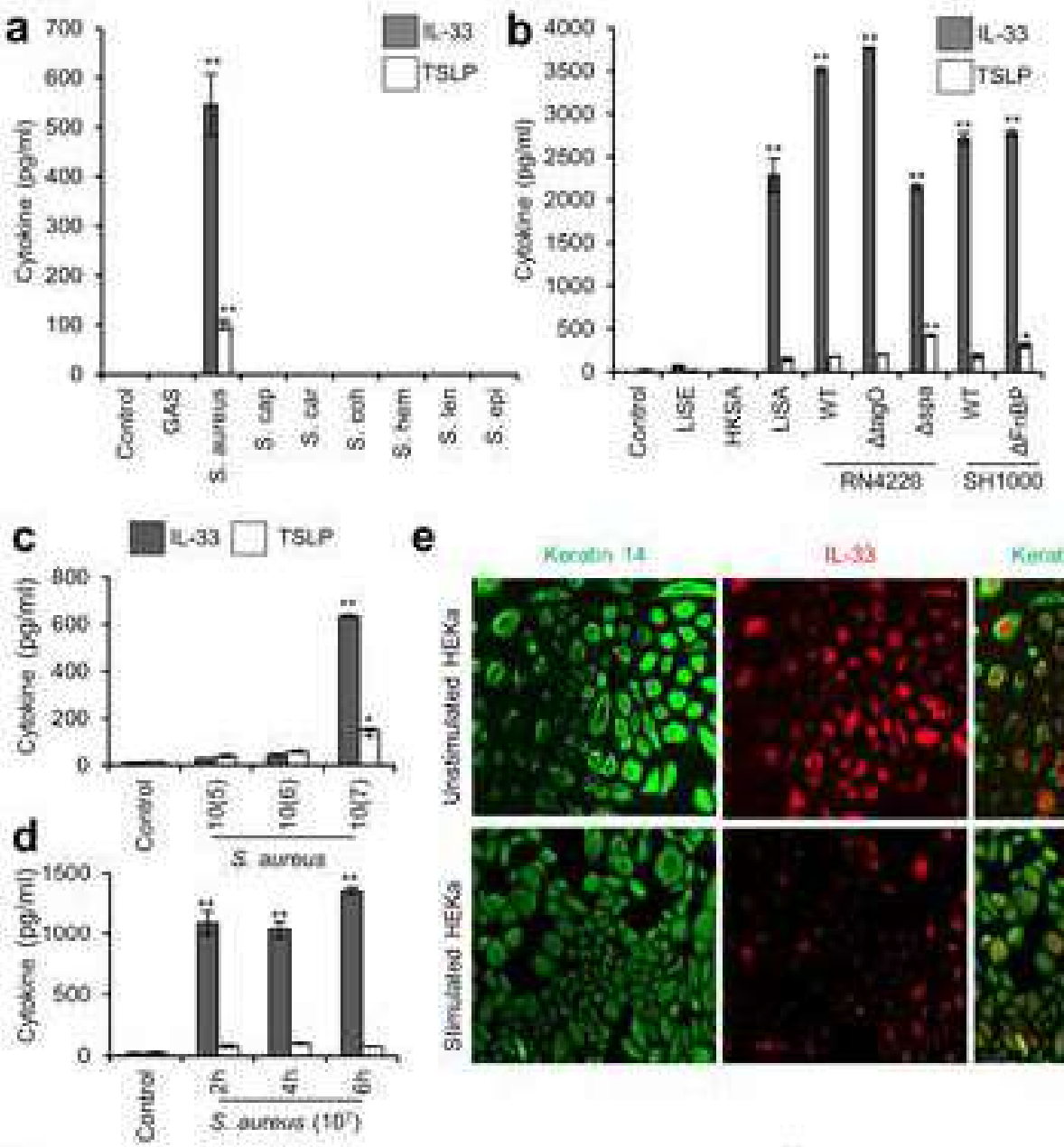

e Koostin in

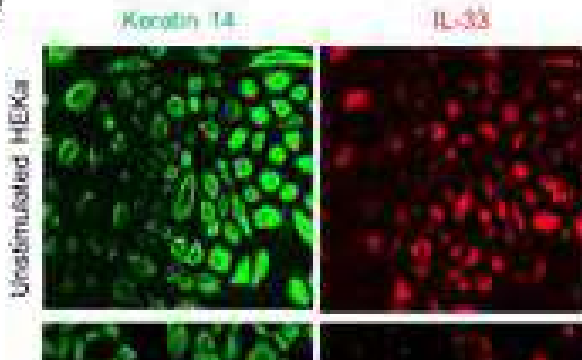

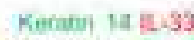

f
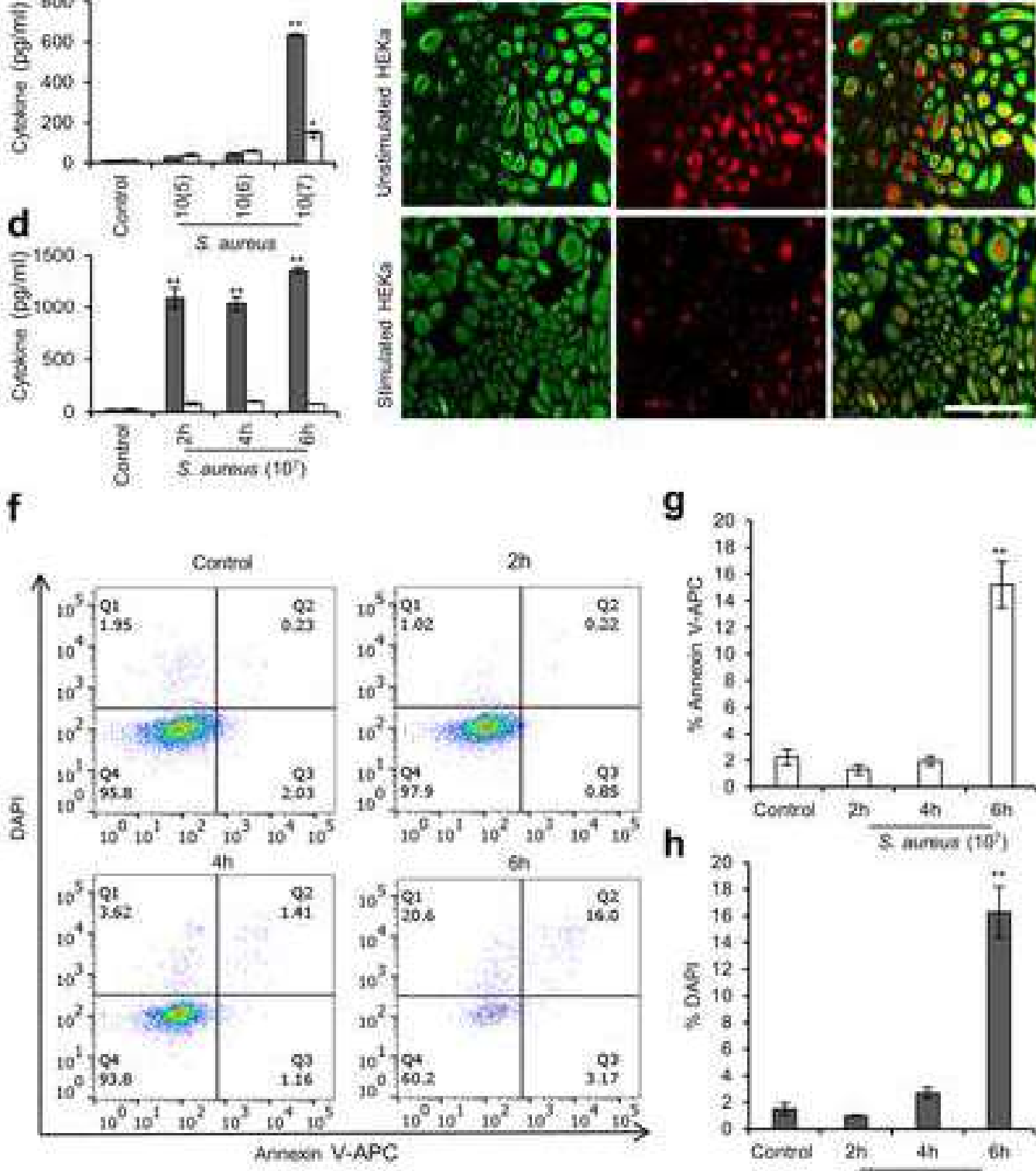

g
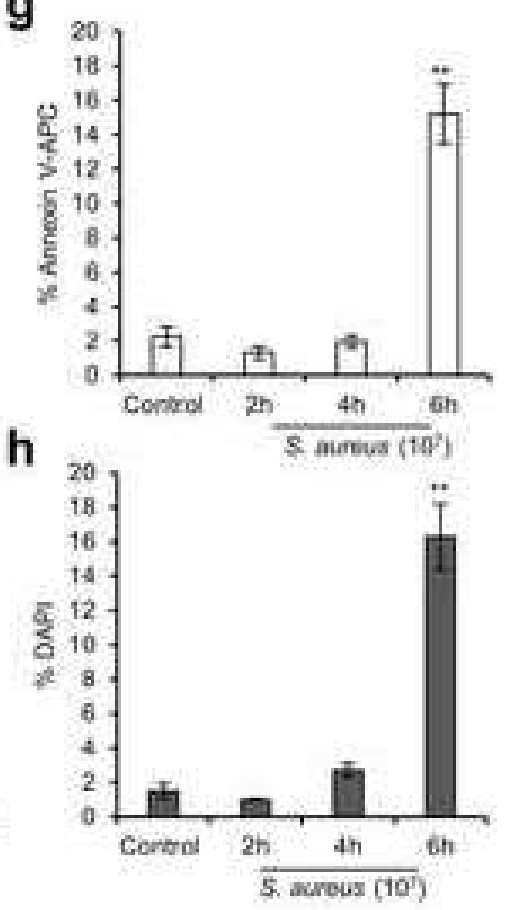

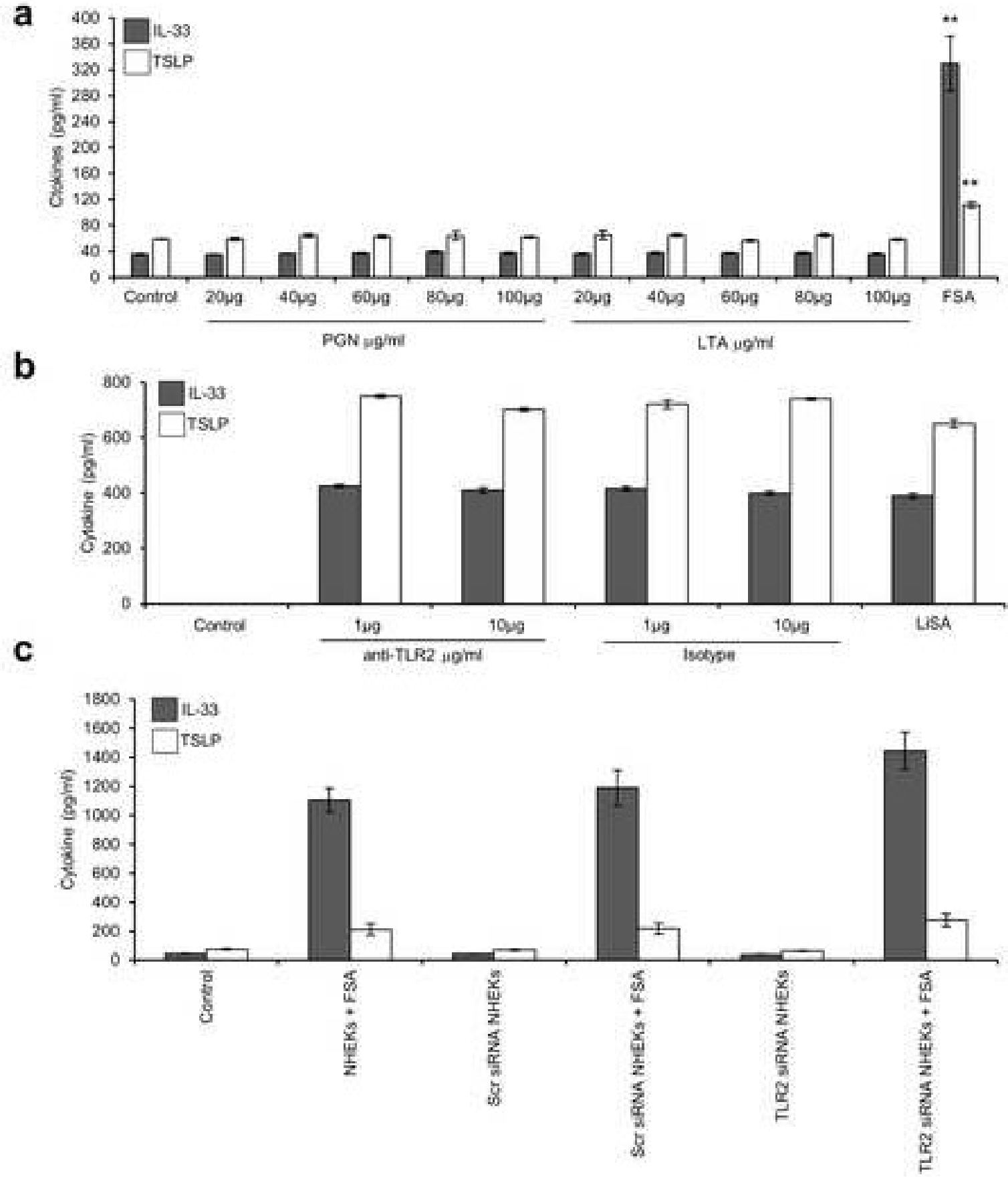
a

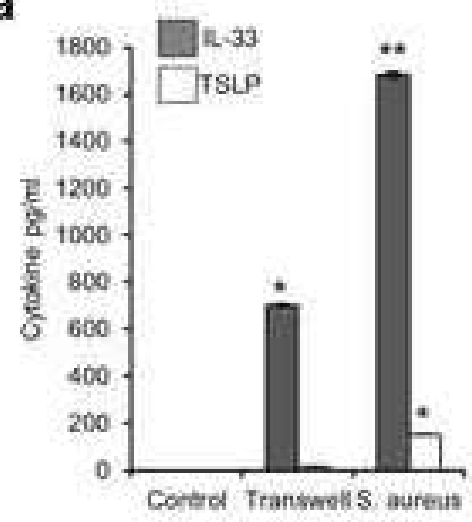

C

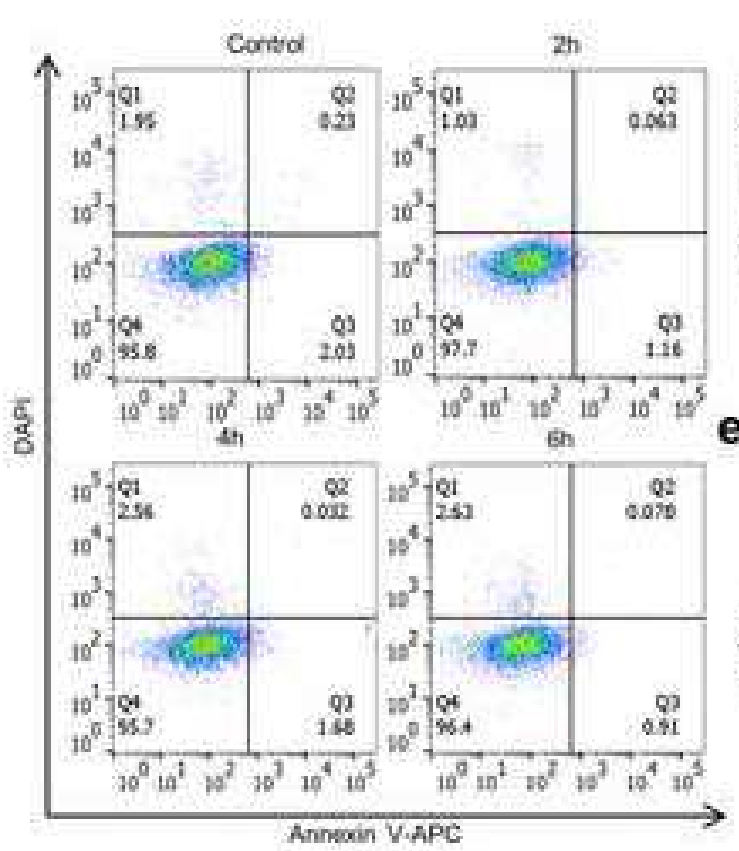

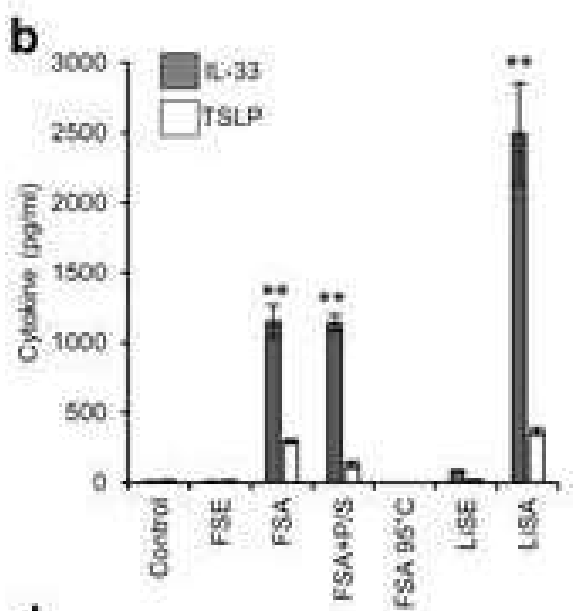

d

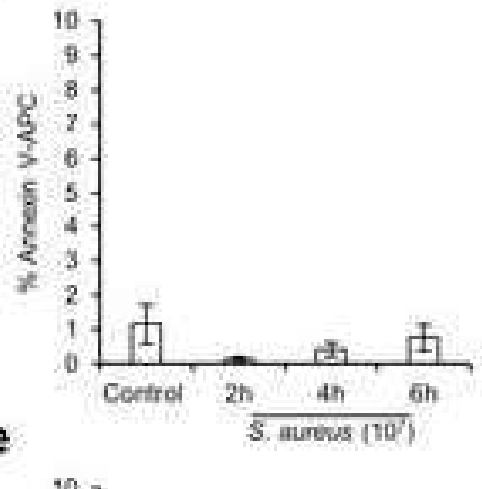

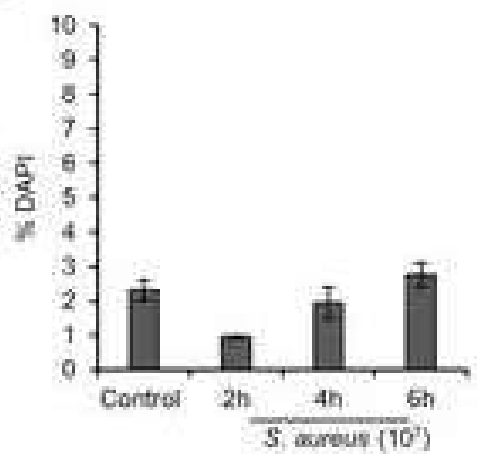



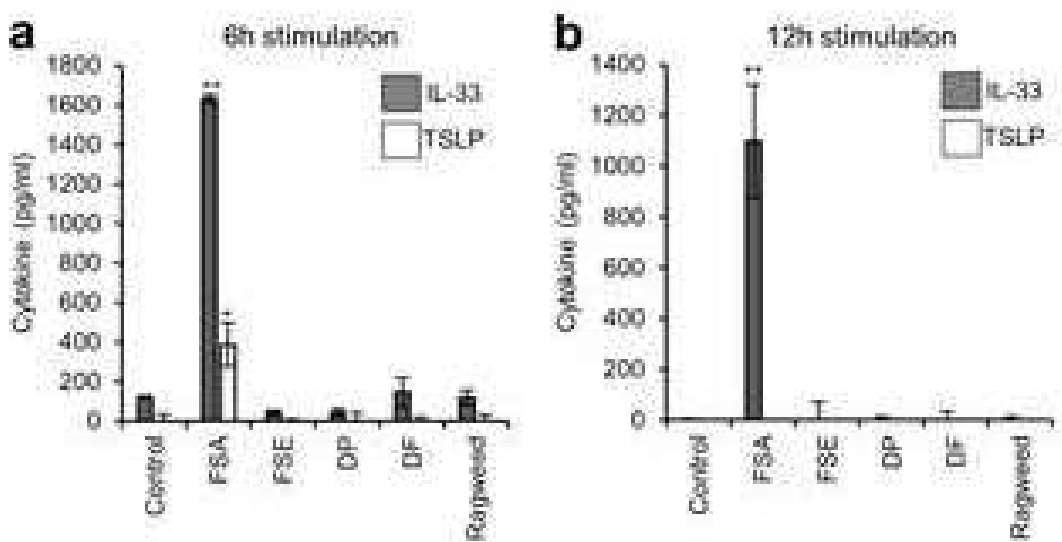

C

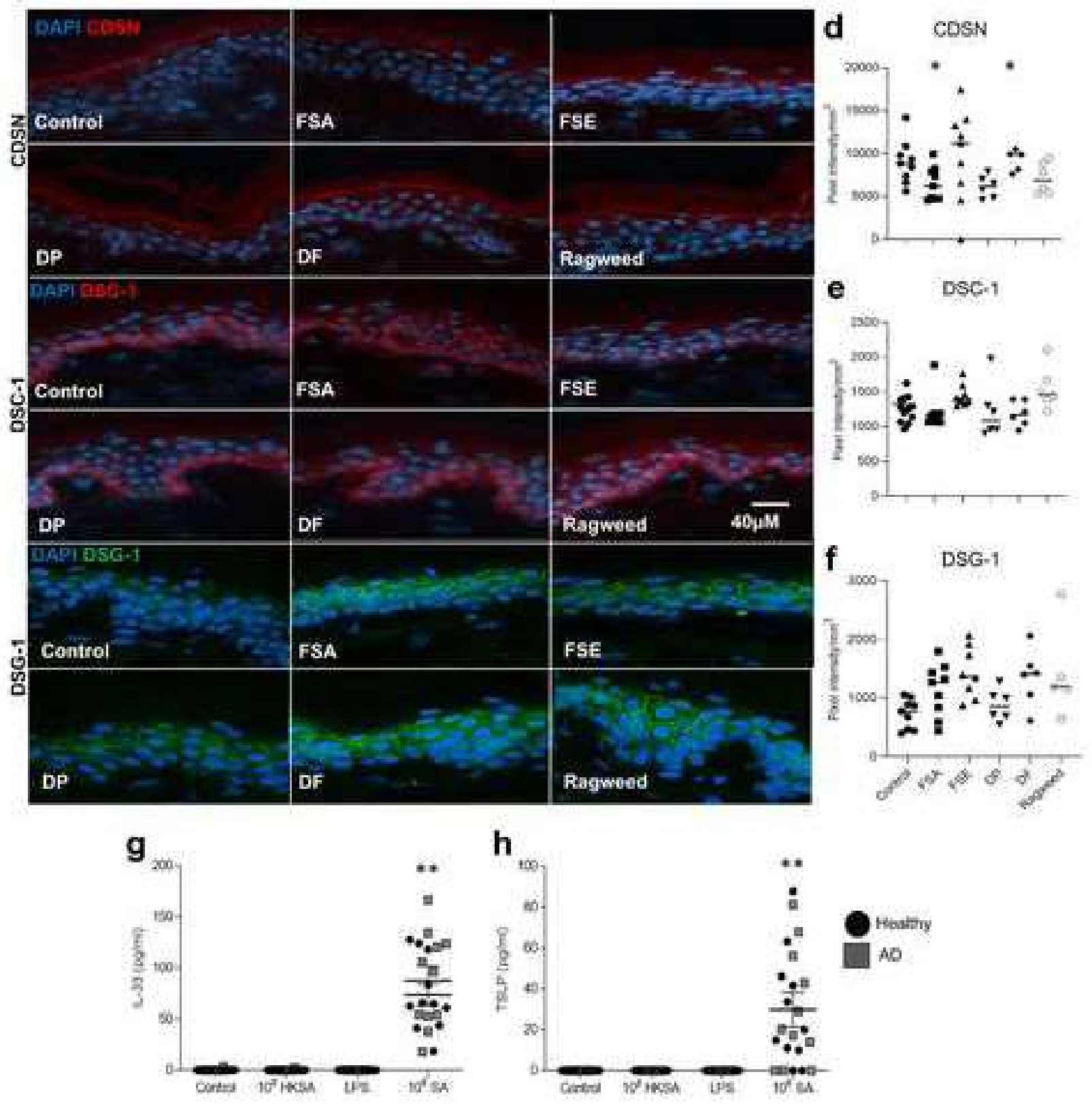




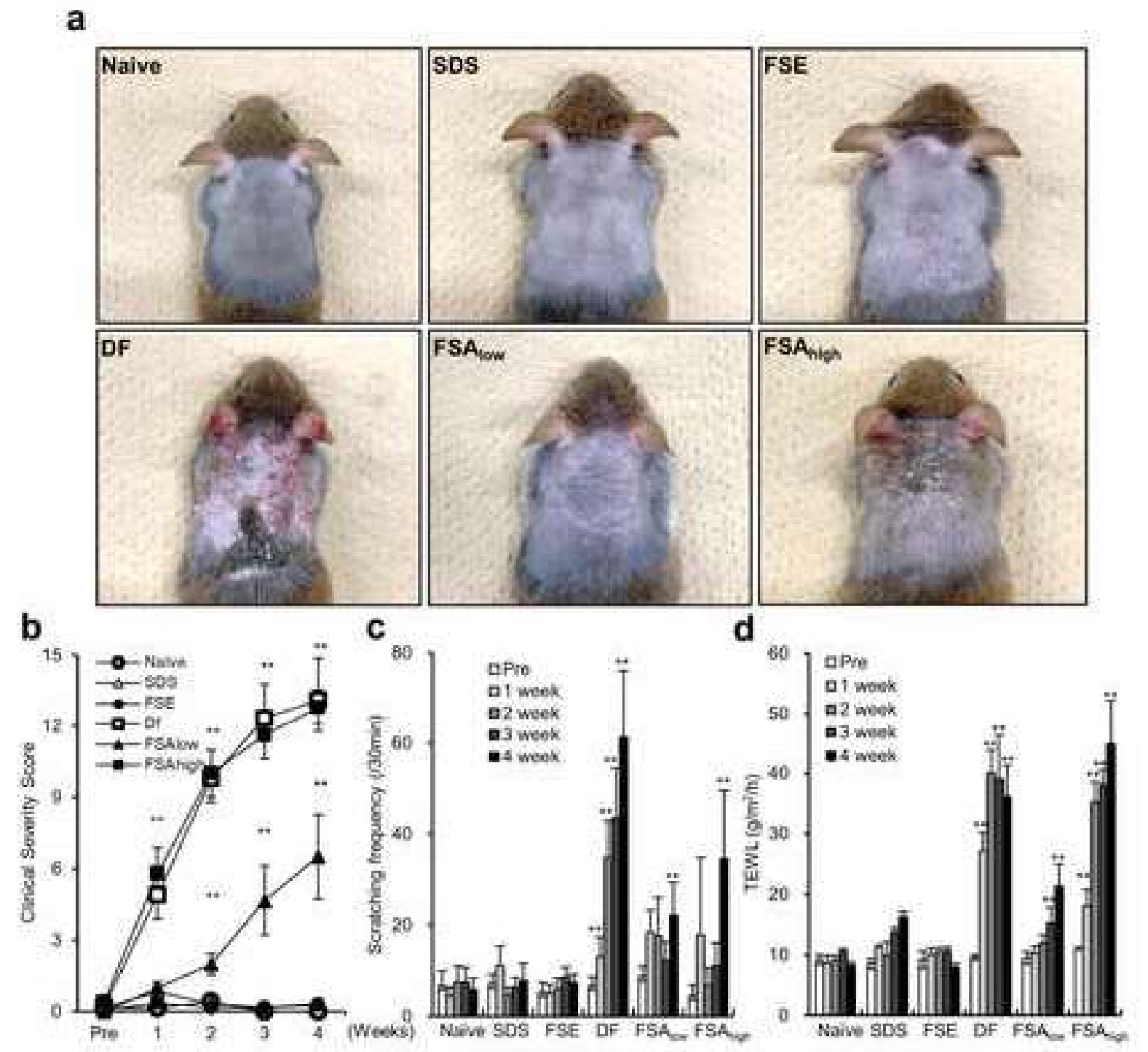




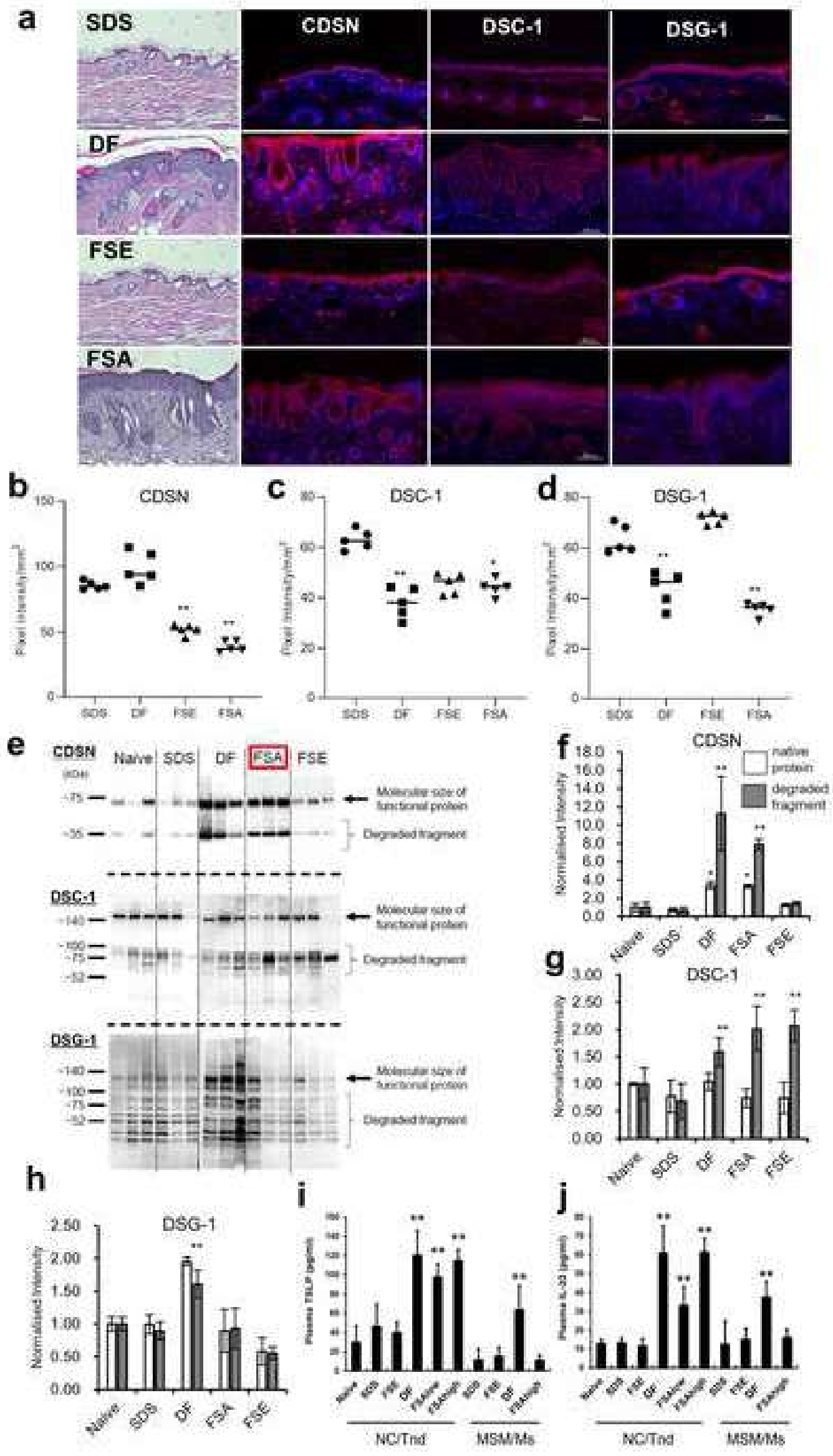




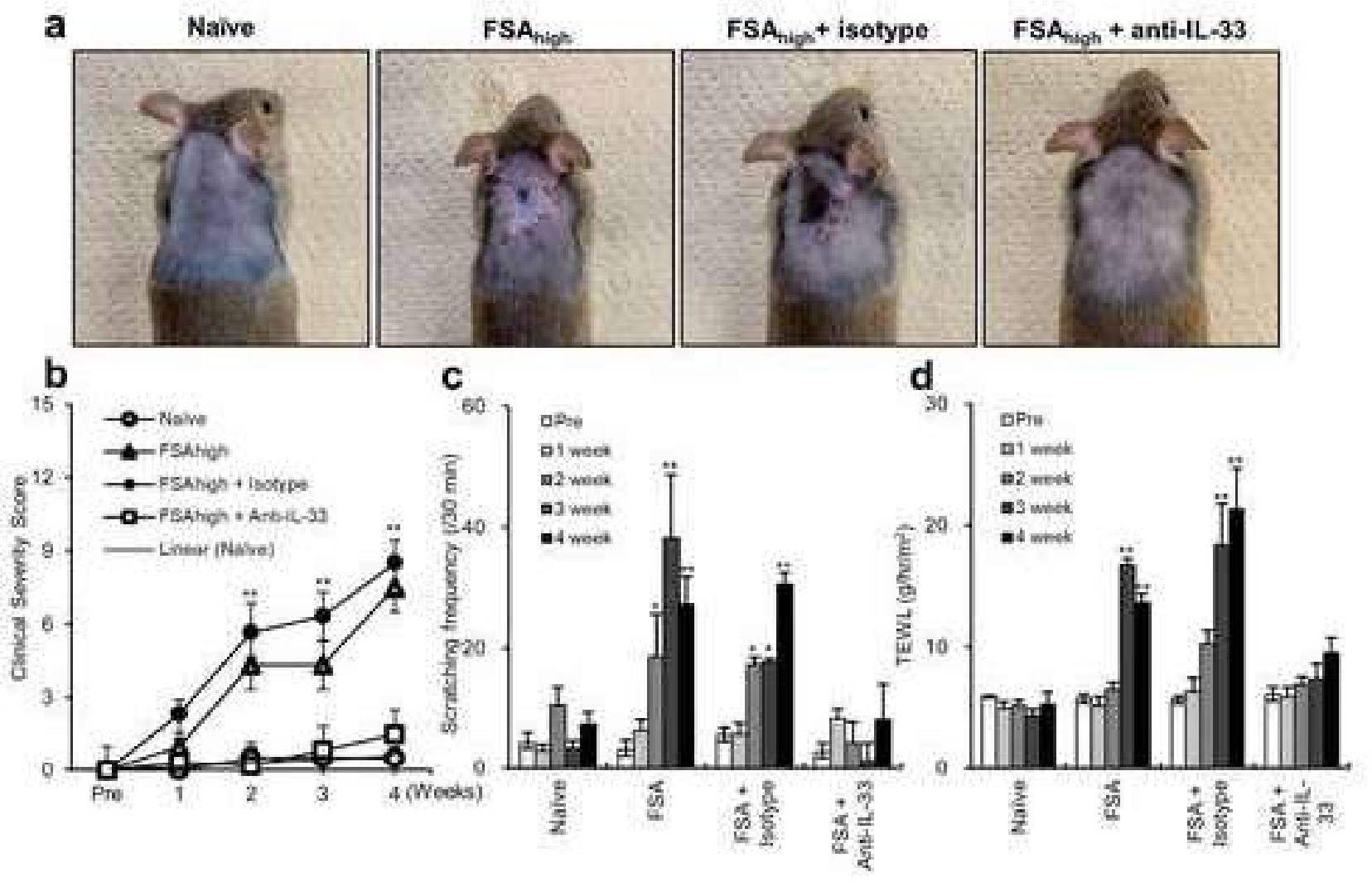




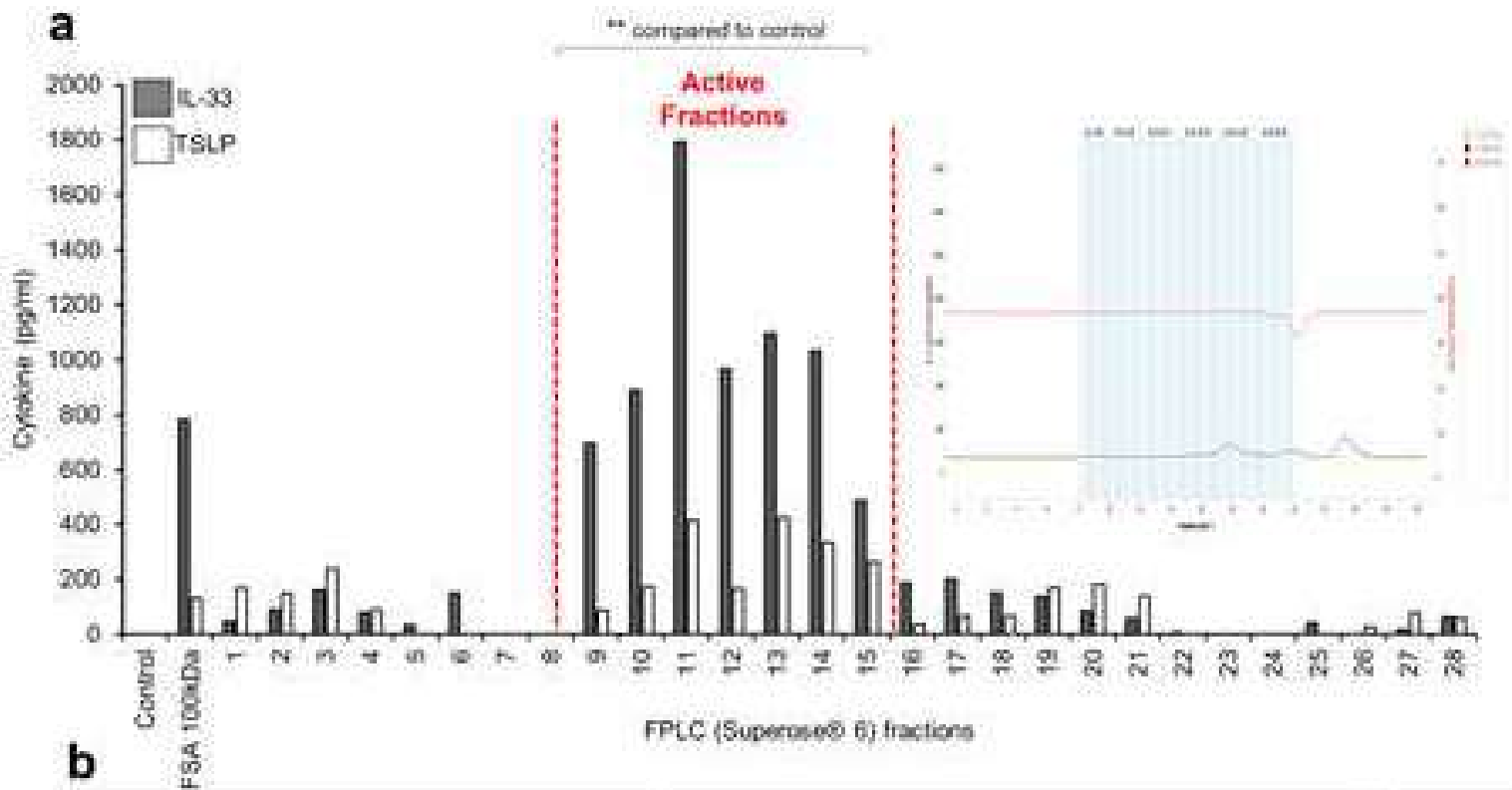

\begin{tabular}{|l|l|c|}
\hline Protein & Function & KDa \\
\hline Dithydrolipoyl dehydrogenase (DLO) & moonlight protein has serine protease activity & 54 \\
\hline S. durous second lgG-binding protein (Sbi) & immunoglobulin binding protein & 50 \\
\hline Pynuate dehydrogenase (PDHa) & calalytic conversion of pyruvate to acesyl-COA $-\mathrm{CO} 2$ & 41 \\
\hline
\end{tabular}

C

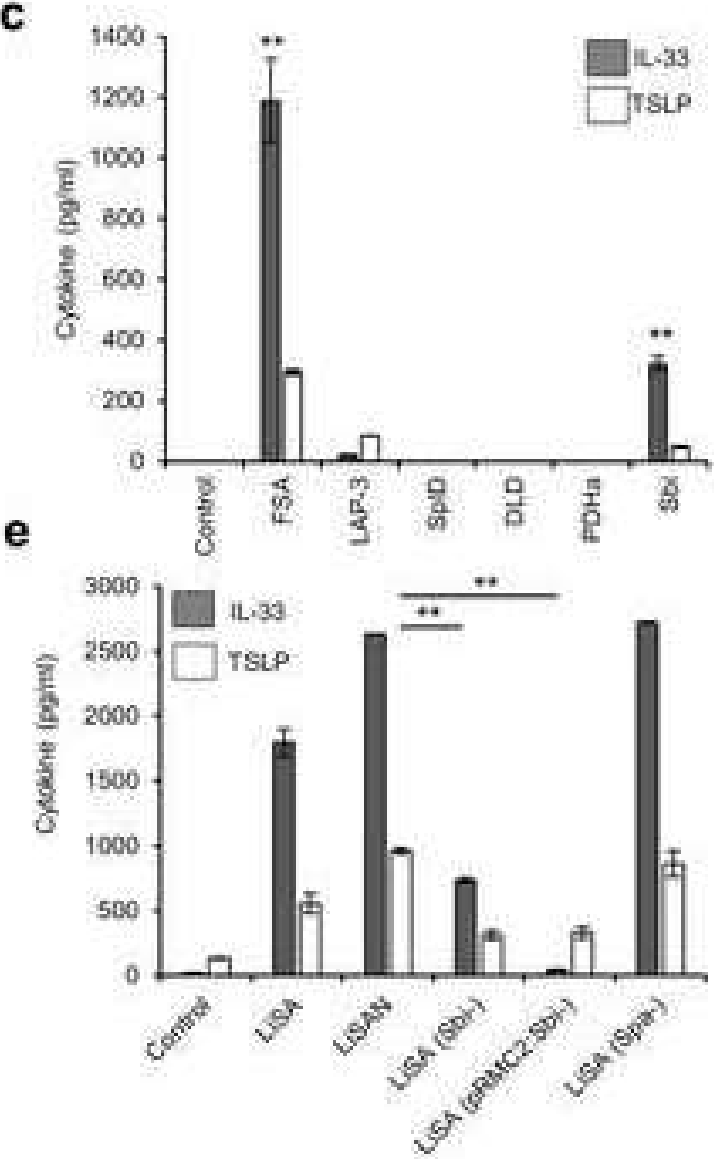

d
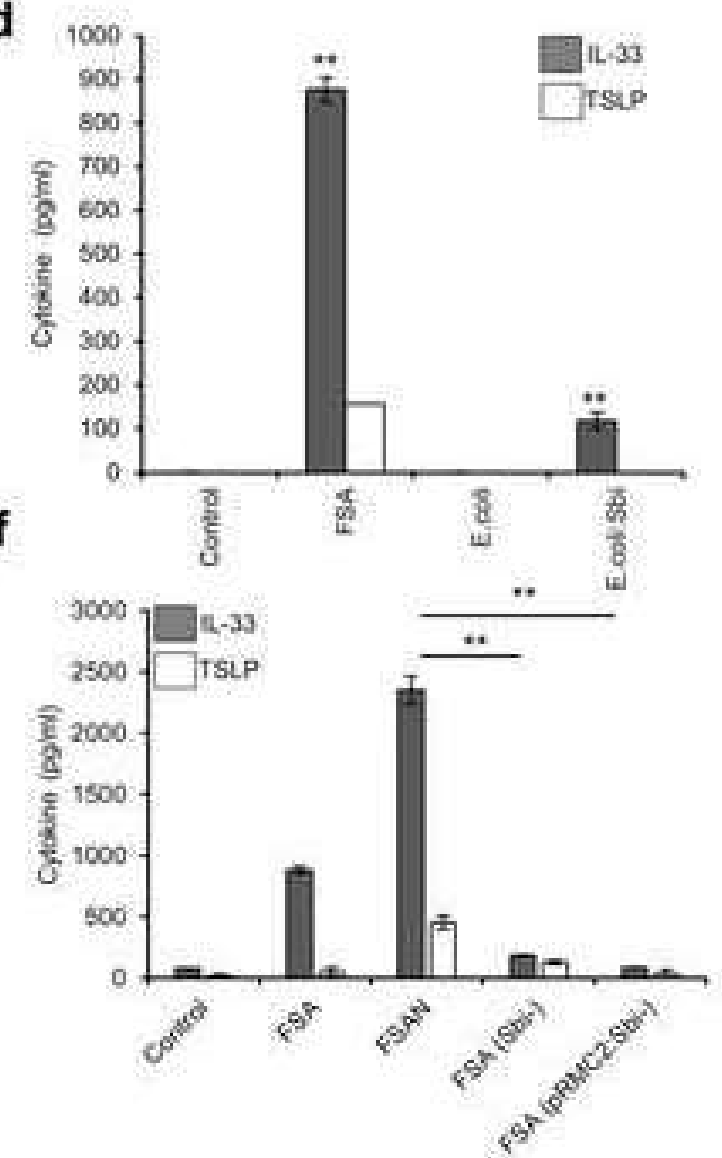


\section{SUPPLEMENTARY ONLINE INFORMATION}

\section{Staphylococcus aureus Second Immunoglobulin-Binding Protein drives atopic dermatitis via IL-33}

Arwa Al Kindi, PhD, ${ }^{1 \dagger}$ Helen Williams, PhD,${ }^{1 \dagger}$ Kenshiro Matsuda, PhD, ${ }^{2}$ Abdullah M. Alkahtani, PhD, ${ }^{3}$ Charis Saville, PhD, ${ }^{1}$ Hayley Bennett, PhD,${ }^{4}$ Yasmine Alshammari, MSc, ${ }^{1}$ Soo Y. Tan, PhD, ${ }^{5}$ Catherine O'Neill, PhD, ${ }^{6}$ Akane Tanaka, DVM, PhD, ${ }^{7}$ Hiroshi Matsuda, DVM, PhD, ${ }^{8}$ Peter D. Arkwright, MD, PhD,${ }^{1 *}$ Joanne L. Pennock, PhD ${ }^{1^{*}}$

${ }^{1}$ Lydia Becker Institute of Immunology and Inflammation, University of Manchester, United Kingdom, ${ }^{2}$ Center for Innovative Drug Discovery, University of Tsukuba, Japan, ${ }^{3}$ Department of Medicine, Microbiology and Parasitology, King Khalid University, Abha, Saudi Arabia, ${ }^{4}$ Genome Editing Unit, University of Manchester, United Kingdom, ${ }^{5}$ National University Health System, Singapore, ${ }^{6}$ Division of Dermatological and Musculoskeletal Sciences, University of Manchester, United Kingdom. ${ }^{7}$ Laboratories of Veterinary Molecular Pathology \& Therapeutics and ${ }^{8}$ Comparative Animal Medicine, Tokyo University of Agriculture \& Technology, Tokyo, Japan

$\dagger, *$ authors contributed equally to this work.

Corresponding author: Dr P D Arkwright, Lydia Becker Institute of Immunology \& Inflammation, University of Manchester, Room 2.21, Core Technology Facility, 46 Grafton St., Manchester, M13 9NT, United Kingdom. Telephone +44 161306 3771, email peter.arkwright@manchester.ac.uk 


\section{Candidate gene cloning and expression}

Sequences of $S$. aureus DLD (gene; pdhD), PDHa (gene; pdhA) and Sbi (gene; sbi) were acquired from KEGG (https://www.genome.jp/kegg/). GST-tagged proteins $p d h D, p d h A$ and $s b i$ genes were cloned into a pGEX plasmid using BamHI and EcoRI restriction enzymes, then transformed into NEB $^{\circledR}$ 5-alpha competent E. coli cells (New England BioLabs, UK) following manufacturer's instructions. Once cloning was verified, $p d h D, p d h A$ and $s b i$ were expressed in BL21 (DE3) E-coli cells (New England BioLabs, UK) for $3 \mathrm{~h}$ at $37^{\circ} \mathrm{C}$ or in Arctic Express E. coli (Agilent Technologies, USA) for $24 \mathrm{~h}$ at $12^{\circ} \mathrm{C}$. After expression, cell cultures were centrifuged at $3,500 \mathrm{~g}$ for $20 \mathrm{~min}$ at $4^{\circ} \mathrm{C}$. Supernatant was discarded and cell pellets were frozen in liquid nitrogen and then at $-80^{\circ} \mathrm{C}$ until used for protein purification by Glutathione Sepharose $4 \mathrm{~B}$ beads.

\section{Cloning and expression of SpID}

Full length SplD was amplified from the clinical isolate of S. aureus and cloned into an ampicillinresistant pQE30 vector. The protein was expressed in chemically competent $E$. coli cells BL21 grown at $37^{\circ} \mathrm{C}$ in $\mathrm{TB}$ (Sigma-Aldrich) containing $100 \mu \mathrm{g} / \mathrm{ml}$ ampicillin, purified using a HiTrap column (GE Healthcare) and eluted with imidazole. 
Table SI List of materials used in this study

REAGENT or

SOURCE

IDENTIFIER

RESOURCE

\section{Bacterial strains}

S. aureus (LiSA)

A. McBain, University of Manchester, UK

MBRG 16.1

S. aureus RN4220 WT

G. Xia, University of Manchester, UK

N/A

S. aureus RN4220; $\Delta$ tagO

G. Xia, University of Manchester, UK

$\mathrm{N} / \mathrm{A}$

S. aureus RN4220; $\Delta$ spa

G. Xia, University of Manchester, UK

N/A

S. aureus $\mathrm{SH} 1000$ and its Joan Geoghegan, University of Dublin, Ireland isogenic fnbA fnbB mutant $8325-4$

$S$. aureus Sbi mutant Joan Geoghegan, University of Dublin, Ireland

N/A

S. aureus pRMC2::Sbi mutant Joan Geoghegan, University of Dublin, Ireland

N/A

S. capitis

G. Xia, University of Manchester, UK

ATCC 27840

S. carnosus

G. Xia, University of Manchester, UK

TM300

S. cohnii

G. Xia, University of Manchester, UK

ATCC 29974

S. hemolyticus

G. Xia, University of Manchester, UK

JCSC 1435

S. lentus

G. Xia, University of Manchester, UK

3472

S. epidermidis

G. Xia, University of Manchester, UK

1457

Streptococcus pyogenes (GAS)

E. coli $\mathrm{DH} 5 \alpha$

A. McBain, University of Manchester, UK

NCTC 12696

E.

E. coli $\mathrm{NEB} \AA$ 5-alpha

UK

N/A

E. coli BL21(DE3)

New England BioLabs, UK

$\mathrm{C} 2987 \mathrm{H}$

E. coli (DE3) Arctic Express

New England BioLabs, UK

$\mathrm{C} 2527 \mathrm{H}$

Agilent Technologies, USA

230192

\section{Human keratinocytes culture}

Primary Normal Human

PromoCell, Heidelberg, Germany

C-12002

Epidermal Keratinocytes

(NHEK)

DetachKit2 Trypsin, Trypsin

PromoCell, Heidelberg, Germany

C-41212

neutralizing solution

Human Epidermal Keratinocytes, ThermoFisher Scientific, UK

C-0055C

(HEKa) 


\begin{tabular}{|c|c|c|}
\hline \multicolumn{3}{|c|}{ Antibodies and fluorescent labelling } \\
\hline $\begin{array}{l}\text { Anti-Human Corneodesmosin } \\
\text { (CDSN) }\end{array}$ & Invitrogen, UK & PA562936 \\
\hline $\begin{array}{l}\text { Anti-Human Desmocollin-1 } \\
\text { (DSC-1) }\end{array}$ & Invitrogen, UK & PA550651 \\
\hline $\begin{array}{l}\text { Anti-Human Desmoglein-1 } \\
\text { (DSG-1) }\end{array}$ & Invitrogen, UK & 326000 \\
\hline $\begin{array}{l}\text { Texas red goat anti-rabbit } \\
\text { antibody }\end{array}$ & Life Technologies, USA & $\mathrm{T}-2767$ \\
\hline $\begin{array}{l}\text { Anti-Mouse Corneodesmosin } \\
\text { (CDSN) }\end{array}$ & Santa Cruz & sc-514845 \\
\hline $\begin{array}{l}\text { Anti-Mouse Desmoglein-1 } \\
\text { (DSG-1) }\end{array}$ & Santa Cruz & sc-23910 \\
\hline $\begin{array}{l}\text { Anti-Mouse Desmocollin-1 } \\
\text { (DSC-1) }\end{array}$ & R\&D systems & MAB7367 \\
\hline Anti-Mouse IL-33 & R\&D systems & AF3626 \\
\hline \multicolumn{3}{|l|}{ Plasmids } \\
\hline pGEM-T easy vector & Promega, UK & A3600 \\
\hline pGEM-T;SplD & This study & - \\
\hline pQE30 & Qiagen, Crawley, UK & - \\
\hline pQE30;SplD & This study & \\
\hline pGEX-6P-1 & Sigma Aldrich, UK & GE28-9546-48 \\
\hline pGEX;pdhA & This study & - \\
\hline pGEX;pdhD & This study & - \\
\hline pGEX;Sbi & This study & - \\
\hline
\end{tabular}


Table SII List of Primer sequences used in this study

\begin{tabular}{|c|c|c|}
\hline Oligonucleotide & Sequence (5'-3') & Purpose \\
\hline SpID BamH1 F & $\begin{array}{l}\text { CCTGTAGGATCCATGAATAAAAATAT } \\
\text { AATCATCAAAAGTATTGCGG }\end{array}$ & \multirow{2}{*}{$\begin{array}{l}\text { Used to amplify SplD from Clin1- } \\
\text { SA for cloning using BamHI- } \\
\text { EcoRI sites of pGEM-T }\end{array}$} \\
\hline SpID HindIII R & $\begin{array}{l}\text { GCGCGATAAGCTTTTATTATTTATCTA } \\
\text { AАTTATCTGCAATAAATTTCTTAAT }\end{array}$ & \\
\hline M13 F & CGCCAGGGTTTTCCCAGTCACGAC & \multirow{2}{*}{$\begin{array}{l}\text { Used to sequence } S p l D \text { insert in } \\
\text { pGEM-T vector }\end{array}$} \\
\hline M13 R & AGCGGATAACAATTTCACACAGGA & \\
\hline PQE30 F & AAGTGCCACCTGACGTCTAAG & \multirow{2}{*}{$\begin{array}{l}\text { Used to sequence } S p l D \text { insert in } \\
\text { pQE30 vector }\end{array}$} \\
\hline PQE30 R & GGAGTTCTGAGGTCATTACTG & \\
\hline pGEX F & GGGCTGGCAAGCCACGTTTGGTG & \multirow{2}{*}{$\begin{array}{l}\text { Used to screen for inserts ( } p d A \text {, } \\
p d h D \text { and } S b i) \text { cloned in pGEX }\end{array}$} \\
\hline pGEX R & CCGGGAGCTGCATGTGTGTCAGAGG & \\
\hline
\end{tabular}

